\title{
An integrative dynamic model of brain energy metabolism using in vivo neurochemical measurements
}

\author{
Mathieu Cloutier • Fiachra B. Bolger • John P. Lowry • \\ Peter Wellstead
}

Received: 5 December 2008 /Revised: 12 March 2009 /Accepted: 19 March 2009

(C) Springer Science + Business Media, LLC 2009

\begin{abstract}
An integrative, systems approach to the modelling of brain energy metabolism is presented. Mechanisms such as glutamate cycling between neurons and astrocytes and glycogen storage in astrocytes have been implemented. A unique feature of the model is its calibration using in vivo data of brain glucose and lactate from freely moving rats under various stimuli. The model has been used to perform simulated perturbation experiments that show that glycogen breakdown in astrocytes is significantly activated during sensory (tail pinch) stimulation. This mechanism provides an additional input of energy substrate during high consumption phases. By way of validation, data from the perfusion of $50 \mu \mathrm{M}$ propranolol in the rat brain was compared with the model outputs. Propranolol affects the glucose dynamics during stimulation, and this was accurately reproduced in the model by a reduction in the glycogen breakdown in astrocytes. The model's predictive capacity was verified by using data from a sensory stimulation (restraint) that was not used for model calibration. Finally, a sensitivity analysis was conducted on the model parameters, this showed that the control of energy
\end{abstract}

Action Editor: Upinder Singh Bhalla

Financial support of Science Foundation Ireland under award 03/RP1/ I382 is gratefully acknowledged.

M. Cloutier $\cdot$ P. Wellstead $(\bowtie)$

Hamilton Institute, National University of Ireland Maynooth,

Maynooth, Ireland

e-mail: peter.wellstead@nuim.ie

F. B. Bolger · J. P. Lowry

Sensors Development Unit, Bioelectroanalysis Laboratory,

Department of Chemistry,

National University of Ireland Maynooth,

Maynooth, Ireland metabolism and transport processes are critical in the metabolic behaviour of cerebral tissue.

Keywords Astrocytes · Brain energy metabolism . Kinetic modelling $\cdot$ Neurons $\cdot$ Systems biology

\section{Introduction}

Brain energy metabolism is central to all cellular processes that maintain neuronal functionality. Naturally, this area has received considerable attention, with conceptual studies spanning the classical view of energy metabolism (glucose oxidation exclusively in neurons) to that proposed by Pellerin and Magistretti (1994) for the astrocyte-neuron lactate shuttle (ANLS). Critical additions to our understanding have been provided by in vivo experimental information from new measurement technologies. In this regard, imaging methods (fMRI, PET) have contributed significantly, while real-time measurement of neurochemical concentrations (Lowry and O'Neill 2006) has provided a source of quantitative information without losing the in vivo context.

In recent years a further potent tool, in the form of mathematical modelling (Aubert and Costalat 2005), has been added to this mix of conceptual studies and experimental measurement. Mathematical modelling allows biological hypotheses to be tested within a computer simulation (in silico) framework that combines conceptual models with experimental data in a quantitative form. Our aim in this article is to add an integrative, systems dimension to this approach with an improved mathematical description of the relevant mechanisms and an explicit systems approach to the modelling of energy metabolism. 


\subsection{Integration via mathematical modelling}

As our knowledge of biological processes become more sophisticated, approaches are required that are correspondingly more systems based and allow the integration of a range of biological phenomena. In the case of brain energy metabolism it can be argued that such an integrative philosophy first appeared in the ANLS hypothesis proposed by Pellerin and Magistretti (1994). In the same spirit, the linkage between cerebral blood flow systems and metabolism is a further area that requires a systems approach that integrates a diversity of knowledge and a range of data sets (see for example Gjedde 2002).

\subsection{In vivo data as a part of the integrative approach}

Mathematical models are credible tools for testing biological hypotheses only when they are calibrated with relevant experimental data. For example, neuroimaging technology offers a family of data sources that have been widely used to provide information on brain metabolism function through the quantification and localization of cerebral activity (Miller 2008). A complementary source of data for local, quantitative, measurements of chemical species in the brain is in vivo electrochemical sensing whereby realtime data can be obtained from freely moving animals submitted to physiological stimuli (Lowry and O'Neil. 2006). With this approach, a microvoltammetric electrode (sensor) is implanted in a specific brain region to monitor local changes in the concentration of energy substrates and other neurochemical species in the extracellular fluid with sub-second time resolution over extended periods.

\subsection{Understanding brain energy metabolism}

within the appropriate analytical framework

Mathematical modelling of biological hypotheses, combined with in-vivo sensing, is especially important for the study of brain energy metabolism. Brain energy metabolism is characterized by interactions and regulation mechanisms that are difficult to unambiguously assess using the limited range of in vivo experimental observations that are possible. A classical method of measuring/estimating metabolic reaction rates such as Flux Balance Analysis (FBA; Varma and Palsson 1994) can be applied. However, this requires flux estimations at the cell boundaries (membranes), and while this is theoretically possible for brain metabolism, the analysis is complicated by the presence of two cellular species (astrocytes and neurons). As a result, more hypotheses are required (see Çakir et al. 2007) such that the analysis becomes correspondingly more complex and less conclusive. A further drawback of FBA is that it is limited to steady-state conditions; this is a major constraint for brain metabolism, as the response to stimulation is intrinsically transient.

Finally, it should be recalled that the important interactions within the brain energy supply system are not limited to traffic between astrocytes, neurons and capillaries. Metabolic regulation is composed of all the possible interactions between biomolecules (genes, enzymes, metabolites, substrates etc.) involved in a metabolic process. These intracellular interactions and their associated subsystems will drive the overall cellular behaviour (i.e. glucose consumption, lactate production) and must be considered in any integrative modelling exercise. Thus, to correctly assess brain energy metabolism, the analytical framework must be able to cope with all three particularities of the cerebral tissue, namely: (i) cell-to-cell interactions through the extracellular space, (ii) transient conditions representative of the cerebral environment (stimulation, changes in blood flow) and (iii) intracellular interactions and regulation that drive the overall tissue metabolic behaviour.

A dynamic, integrative approach (such as presented in this study) has the advantage of being a good abstraction of the system, given our current knowledge and in vivo data sources. It imposes minimal biological hypotheses, with the idea of capturing the most important and relevant dynamic features of cerebral energy metabolism. The calibrated model then becomes a repository of known mechanisms based on realistic quantitative experimental observations. As a result it can plausibly be presented as an analytical framework for the study of brain energy metabolism.

\subsection{Contributions of this study}

There is significant prior work on the mathematical modelling of brain metabolism. In particular, Aubert and co-workers have recently published compartmented models for brain energy metabolism that allowed, amongst other things, the ANLS to be studied from a theoretical point of view (Aubert and Costalat 2005; Aubert et al. 2005). Using these models as a starting point, we have developed an integrative, dynamic, metabolic model for brain energy metabolism that has significant novel aspects. Specifically, the model presented here includes the following additions or modifications when compared to the model by Aubert and Costalat (2005):

- The coordination of neuronal and astrocytic activity is considered through neurotransmitter (glutamate) cycling.

- The central energy metabolism is expanded by considering 5 reactions for glycolysis instead of 3 and mitochondrial regulation has been modified to include inhibition at high energetic states. 
- The glycogen dynamics in astrocytes are included. This is crucial in providing a buffering system for the 'energy budget' between high and low activity periods.

- The calibration of kinetic parameters for metabolic pathway regulation (reaction rates, affinity constant, regulation constants etc.) is performed using in vivo real-time neurochemical measurements of glucose (GLC) and lactate (LAC) from freely moving animals during stimulation (Fillenz and Lowry 1998; Bolger et al. 2006). As such the model presented here is as close as possible (using current technology) to the dynamic performance of energy metabolism in the brain.

- The results of a sensitivity analysis of the calibrated model are given. This allows the identification of functional properties of the brain tissue that are critical to energy metabolism.

\section{Model description}

A complete description of the model and all its properties (states, mass balances and kinetic equations) is presented in
Appendix A. This section is therefore restricted to an overview of the model and its sub-systems. Referring to the schematic interpretation of the model shown in Fig. 1, we begin by considering the various model component systems.

\subsection{Cerebral compartments and exchange systems}

Four compartments are considered: neurons (variables indexed ' $\mathrm{n}$ '), astrocytes (variables indexed ' $\mathrm{g}$ '), capillaries (variables indexed 'c') and the extracellular space (variables indexed ' $\mathrm{e}$ '). Within this compartmental arrangement, GLC is transferred from capillaries to neurons and astrocytes via the extracellular space (see Fig. 1, and the variables, $v_{\mathrm{GLC}}{ }^{\mathrm{ce}}, v_{\mathrm{GLC}}{ }^{\text {en }}$ and $v_{\mathrm{GLC}}{ }^{\text {eg }}$ in Fig. A-1, Appendix A.1). In addition, a direct transfer of GLC from capillaries to astrocytes is considered $\left(v_{\mathrm{GLC}}{ }^{\mathrm{cg}}\right)$. Transporters for GLC are described by facilitated diffusion (see Table A.1.2 in Appendix A.1). LAC transport $\left(\nu_{\mathrm{LAC}}{ }^{\mathrm{ec}},{\nu_{\mathrm{LAC}}}^{\text {ne }}, v_{\mathrm{LAC}}{ }^{\text {ge }}\right.$ and $v_{\text {LAC }}{ }^{\text {gc }}$; see Appendix A.1) is described with the same mechanisms as for GLC, with the difference that the gradient will favour release of LAC (instead of uptake in the case of GLC). Oxygen $\left(\mathrm{O}_{2}\right)$ and carbon dioxide $\left(\mathrm{CO}_{2}\right)$

\section{Extracellular volume}

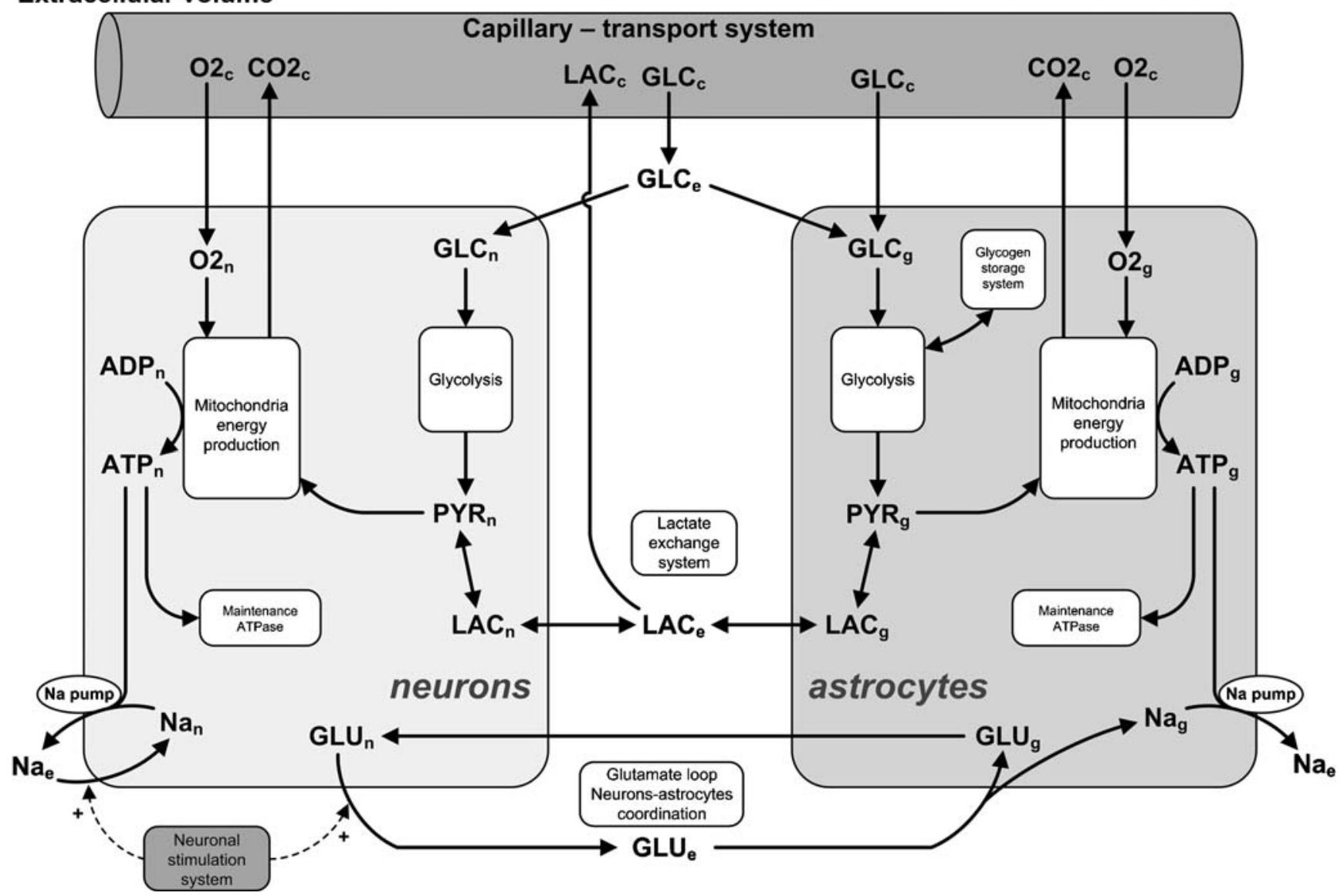

Fig. 1 Schematic view of the model for astrocytes-neurons metabolism. Fluxes names refer to reactions in Appendix A 
dynamics in the extracellular space are neglected. As regards $\mathrm{O}_{2}$, the intracellular dynamic is considered, with a balance between transport (Figure A.5: $\mathrm{v}_{\mathrm{O} 2}{ }^{\mathrm{cn}}, \mathrm{v}_{\mathrm{O} 2}{ }^{\mathrm{cg}}$ ) and consumption (Figure A.5: $\mathrm{v}_{\text {mito }}{ }^{\mathrm{n}}, \mathrm{v}_{\text {mito }}{ }^{\mathrm{g}}$ ).

\subsection{Energy metabolism}

Within the glycolysis systems in Fig. 1, GLC entering neurons or astrocytes is converted to pyruvate (PYR) in five steps. The details of these steps are given in Appendix A.2 and described here in overview form. The glycolysis model describes adenosine triphosphate (ATP) consumption by the hexokinase $\left(\nu_{\mathrm{HK}}\right)$ and phosphofructokinase $\left(\nu_{\mathrm{PFK}}\right)$. Glyceraldehyde-3-P (GAP) produced from the phosphofructokinase (PFK) reaction is then converted to phosphoenolpyruvate (PEP) with NADH (nicotinamide dinucleotide) regeneration from NAD $\left(\nu_{\mathrm{PGK}}\right)$ and, finally, PYR is produced from PEP by the pyruvate kinase $\left(\nu_{\mathrm{PK}}\right)$. PYR can either be converted to LAC $\left(v_{\mathrm{LDH}}\right)$ or oxidized in the mitochondria $\left(v_{\text {mito }}\right)$ to regenerate the main energy 'currency' in the cerebral tissue, ATP, from ADP (adenosine diphosphate).

In general, the regulation mechanisms and reactions for energy metabolism are based on the work of Aubert and Costalat (2005), Gjedde (1997) and Heinrich and Schuster (1996). The addition of hexokinase reaction (not considered by previous studies by Aubert and Costalat) allows an additional layer of control on the GLC consumption by the cerebral tissue, this addition improves the description of local increases in GLC consumption upon activation. In our model, mitochondrial regulation was modified from Aubert and Costalat (2005) to include inhibition of mitochondrial activity at high ATP/ADP ratio (Gjedde 1997). Thus, the model describes mitochondrial regulation from the availability of PYR, $\mathrm{O}_{2}$ and the energetic requirements of the tissue. Energetic metabolism in neurons and astrocytes is buffered by phosphocreatine ( $\mathrm{PCr}$ ) which is used to regenerate ATP during short-term abrupt increases in energy demand. Adenosine monophoshphate (AMP) equilibrium with ADP and ATP through the adenylate kinase reaction is also considered, using parameters from Aubert and Costalat (2005).

\subsection{Neuronal stimulation system}

Neuronal function through stimulation is described in detail in Appendix A.3. In the model described here, the major 'sink' for energy metabolism is the maintenance of ionic gradients in neurons and astrocytes $\left(v_{\text {pump }}\right)$. The dynamics of sodium (Na) are considered, which allows the description of the tissue energetic 'load' both in resting conditions (Na-ATPases pumps working to maintain Na gradient) and during stimulation (increased Na inflow in neurons, $\mathrm{v}_{\text {stim }}{ }^{\mathrm{n}}$ ). The stimulation of neurons (see equation 2 in Table A.3.2) is modelled as in Aubert and Costalat (2005), with a base stimulation rate $\left(\mathrm{v}_{1}{ }^{\mathrm{n}}\right.$ : flow of $\mathrm{Na}$ after neuronal habituation) and a spiking at the onset of stimulation $\left(\mathrm{v}_{2}{ }^{\mathrm{n}}\right)$.

An important phenomenon in brain physiology is the coordination of neuronal and astrocytic response during stimulation. As proposed by Pellerin and Magistretti (1994), a suitable coordination mechanism is the uptake of glutamate (GLU) by astrocytes (with $\mathrm{Na}$ co-transport) after neuronal stimulation, thus forming a loop between neurons and astrocytes. Subsequent to Pellerin and Magistretti's proposal the function of glutamate cycling has been the object of quantitative measurements using ${ }^{13} \mathrm{C}$ NMR by several teams (Shen et al. 1999; Zwingmann and Butterworth 2005; Shestov et al. 2007). The quantitative importance of glutamate cycling was also emphasised in a review by Hyder et al. (2006) and in modelling studies (Gruetter et al. 2001; Uffmann and Gruetter 2007). In order to obtain a better assessment of the physiological response of the cerebral tissue, the reactions for glutamate cycling are included in the model presented here. As shown in Fig. 1 (with further description in Appendix A.3), this neurotransmitter cycling is described by three reactions: (i) release of GLU by neurons, (ii) cleaning of extracellular space by astrocytes (with $\mathrm{Na}$ co-transport) and (iii) nonstimulatory transfer of GLU back to neurons for further usage in the loop. Note, however, that in our model, the cycling is simplified by neglecting the dynamics of glutamine (GLN) which acts as the non-stimulatory intermediate in the transfer from astrocytes to neurons. The conversion of GLU to GLN in astrocytes, with transfer to neurons and re-conversion to GLU is modelled as one reaction (see Appendix A.3: $\mathrm{v}_{\mathrm{GLU}}{ }^{\mathrm{gn}}$ ). Possible efflux or influx of additional neurotransmitter is neglected in the model, as the current study is focused on energy metabolism. But future improvements on modelling the links between energy metabolism and brain function should include the exchanges between GLU and the intermediates of the TCA cycle in neurons and astrocytes, as it was shown to be significant in certain conditions (Gruetter et al. 2001).

Glutamate cycling activates astrocytic metabolism through two mechanisms. First, Na pumps are activated to maintain the $\mathrm{Na}$ gradient in the astrocytes, thereby consuming ATP. Secondly, the conversion of GLU to GLN ( $v_{\mathrm{GLU}}{ }^{\mathrm{gn}}$ in our model) requires 1 molecule of ATP per molecule of GLU processed. Thus, by considering GLU cycling, the astrocyte-neuron coordinated metabolic activation can be described by a physiologically realistic mechanism, without relying on artificially induced stimulation in astrocytes, as was the case in Aubert and Costalat (2005). 


\subsection{Glycogen metabolism}

The inclusion of astrocytic glycogen (GLY) dynamics in the model (see Appendix A.4) was found to be a key mechanism in describing the in vivo experimental measurements. In the present work, the description of GLY dynamics is limited to two reactions (synthesis and breakdown, see Table A.4.1 in Appendix A.4) with an activation of GLY breakdown during stimulation periods (which could be induced by noradrenaline). This mechanism allows an additional input of energy substrate during high demand periods, an addition that was critical in correctly describing in-vivo experimental observations. As reported by Brown et al. (2005) for optic nerve preparation, astrocytes GLY contributes in maintaining neuronal functionality under aglycemia or during intense stimulation. Here we observe through modelling that this mechanism of GLY storage and reutilization might be contributing significantly (as was also suggested by Forsyth (1996)) to energy metabolism even in normal, physiological stimulations. Further analysis of GLY dynamics might however be required, as it changes the assessment of the dynamics of GLC and $\mathrm{O}_{2}$ consumption and uncoupling phenomenon (GLY is a buffer between the entering GLC flux and its mitochondrial oxidation). As is observed in our simulations, the required influx of GLY to maintain metabolic fluxes during stimulation was coherent with the current estimations of astrocytic GLY content (see Results and Discussion). However, the quantitative relationship between GLY breakdown and the type and intensity of stimulation would require further studies, as the current work is developed only for sensory stimulation.

\subsection{The complete model}

A complete diagram of the model, state variables, fluxes and interactions is presented in Appendix A.5. The model describes 13 independent states for neurons, 14 for astrocytes, 3 extracellular states and 4 capillary states. In total, 44 kinetic equations are used to describe the metabolic system, and the model has 63 kinetic parameters (reaction constants, maximum reaction rates, affinity constants and regulation parameters). These parameters were adjusted and finally determined through a model calibration procedure based upon parameter estimation methods which adjust parameters to optimally match model outputs to observed data (see Model Simulations and Calibration and Appendix B for further details).

The kinetic rates included in the model also use 26 physical constants (volumes fractions, arterial concentrations, electrochemical constants etc.). These constants are assumed to be known and representative of the system (see Aubert and Costalat 2005; Gjedde 2002). A full list of parameters values and physical constants is provided in Appendix A.6. Finally, the input stimulation is described by 9 parameters (strength, duration, CBF fractional increase, etc.). These parameters and their numerical values are given in Table A.6.3 in Appendix A.6.

\section{In vivo data on brain energy metabolism}

The use of time course information for GLC and LAC measurements obtained using microelectrochemical sensors during perturbation experiments with animals provides an insight into the dynamic properties of cerebral metabolism. These time histories can be used to calibrate the model parameters by a parameter estimation procedure. In our case, in vivo data for extracellular cerebral GLC and LAC recorded in freely moving rats was used to perform the calibration. Two different physiological stimulations were considered: tail pinch (TP) and restraint (holding) experiments. Quantitative GLC data from 5 min. TP experiments was taken from Fillenz and Lowry (1998). This data set was complemented with LAC data for $5 \mathrm{~min}$. TP, also from freely moving rats (Bolger et al. 2006). A set of $5 \mathrm{~min}$. restraint (holding) experiments was also considered with LAC measurements (Bolger et al. 2006).

\section{Model simulations and calibration}

The 34 differential equations that constitute the mathematical model, and given in Appendix A, were implemented, solved and analyzed using the Matlab scientific computing environment and the Systems Biology toolbox (SBtoolbox: Schmidt and Jirstrand 2006). The parameters for the model (as listed in Appendix A.6) were determined using both data from the literature and by direct estimation, as follows:

4.1 Parameters, coefficients and physical constant from the literature

The volumes fractions, resting steady-state values, sodium transport parameters and CBF values used in our model were considered to be the same as reported in Aubert and Costalat (2005). For the in vivo parameter estimation/ calibration routines, the initial values for parameters were taken from Aubert and Costalat (2005), where possible. The glutamate loop parameters were first set to produce a cycling of GLU consistent with values reported in the literature by Hyder et al. (2006) and Gjedde (2001). Further data and parameters (affinity constants and maximum reaction rates) were taken from Simpson et al. (2007) for LAC transport and from Barros et al. (2007) for GLC transport. These values for kinetic parameters placed the 
model coefficients in a feasible area of parameter space, and allowed it to operate with physiologically plausible values of concentrations and fluxes. These initial conditions were used as starting points for the estimation routines that calculate the model parameters that provide the best fit to actual experimental data.

\subsection{Parameter estimation routines}

In broad terms, parameter estimation routines work by minimizing the difference between model simulations and the corresponding experimental data. This consists in minimizing the normalized residual sum of squares between simulated and experimental time profiles by changing model parameters. This was done using the parameter estimation routines of the SBtoolbox, in particular the algorithm SBPDparamestimation.m. The experimental data used in here can be separated in two classes:

I. Typical steady-state values (fluxes and concentrations) reported in the literature on brain physiology and

II. In vivo time-series data from perturbation experiments ( 5 min. tail pinch) in freely moving animals.

Appendix B presents typical results of model calibration with resting steady-state values for concentrations and fluxes taken from the literature. These results show that the model is able to correctly describe the overall consumption rates and ratio of GLC, LAC and $\mathrm{O}_{2}$ in the brain (see Appendix B for further details).

Finally, using extracellular GLC and LAC data from tail pinch experiments, it was possible to find a unique set of parameters to describe cerebral metabolic behaviour (see Appendix A.6 for a list of parameters). The only parameters that are allowed to change between different experiments are the stimulation parameters (strength and duration), however this is expected, since different stimulations were used (TP and restraints) and the actual manipulation of animals for in vivo measurements induces an additional variability in the stimulus. The final result of model calibration is a unique set of parameters that describes both the steady-state (resting) and dynamic response of brain metabolism to various physiological stimuli.

\section{Results and discussion}

5.1 Tail pinch experiments: energy, GLC and LAC dynamics

Figure 2 presents an overview of typical simulation results for a $5 \mathrm{~min}$. TP experiment, together with a comparison with experimental in vivo GLC and LAC data. The baseline GLC concentration is correctly simulated, and we note that the baseline $(0.34 \mathrm{mM})$ is relatively low compared to other reports $(0.7-2 \mathrm{mM})$. However, variations are reported between brain regions (McNay et al. 2001) and lower values are also recorded in non-anesthetized animals (McNay and Gold 1999). The calibrated model correctly describes the initial dip in extracellular GLC (Fig. 2f), followed by a post-stimulation overshoot. As mentioned in Fillenz and Lowry (1998), this overshoot is not explained by a decrease in GLC consumption or by the effect of CBF. Also from Fig. 2, the simulated fluxes for glycolysis in astrocytes show an increase in glycolytic rate during stimulation (Fig. $2 \mathrm{~d} \mathrm{v}_{\mathrm{PFK}}{ }^{\mathrm{g}}$ ); this is coherent with the actual literature on brain metabolism, as glycolysis in astrocytes is activated upon neuronal stimulation (Pellerin and Magistretti 1994; Hyder et al. 2006). The simulated decrease in astrocytic ATP concentration (Fig. 2b: ATP $_{\mathrm{g}}$ ) shows how the glutamate loop mechanism affects energy metabolism in astrocytes in a way that is coordinated with neuronal activation. The ATP profile in neurons (Fig. 2b: $\operatorname{ATP}_{n}$ ) showed little variations, as the mitochondrial regulation in neurons allows a much sharper increase in activity upon stimulation (Fig. 2h: $\mathrm{v}_{\text {mito }}{ }^{\mathrm{n}}$ ). This is possible because, among other things, the maximal reaction rate of mitochondrial oxidation is higher in neurons than in astrocytes (see Table A-6 in Appendix A: $\mathrm{v}_{\text {max,mito }}{ }^{{ }}>\mathrm{v}_{\max \text {,mito }}{ }^{\mathrm{g}}$ ), thus matching the Na-ATPase activation in neurons with a sufficient and fast energy supply.

The LAC measurement during TP (see Fig. 2i) shows a rapid increase in concentration after stimulation. This LAC can only originate from an increase in overall production by the cerebral tissue. In the example simulation presented in Fig. 2, the increase in extracellular LAC after stimulation is caused by an increase in LAC production by astrocytes (see $v_{\mathrm{LDH}}{ }^{\mathrm{g}}$ in Fig. $2 \mathrm{~g}$ ).

\subsection{The role of glycogen}

It is clear from the above results that an increase in energy substrate inflow is required to explain how LAC and GLC concentrations could both be higher than their baseline values for a long period of time (post stimulation). The transfer of GLC between capillary and extracellular space (see $\mathrm{v}_{\mathrm{GLC}}{ }^{\mathrm{ce}}$ in Fig. 2c), even though it constitutes the major GLC inflow to the tissue, does not show sufficient variations to explain the GLC profile. As was explained previously (see Model Description and Appendix A.4), the additional inflow during stimulation is assumed to be coming from an activation of glycogen breakdown by a molecular signal (noradrenaline) that is known to be involved in sensory response.

The amplitude of the glycogen (GYL) breakdown flux during TP (see $\nu_{\mathrm{PYG}^{\mathrm{g}}}{ }^{\mathrm{g}}$ in Fig. 2e) was found by the parameter calibration routine to $\mathrm{be} \approx 2 \times 10^{-3} \mathrm{mM} \cdot \mathrm{s}^{-1}$. In 


\section{Tail pinch experiment}
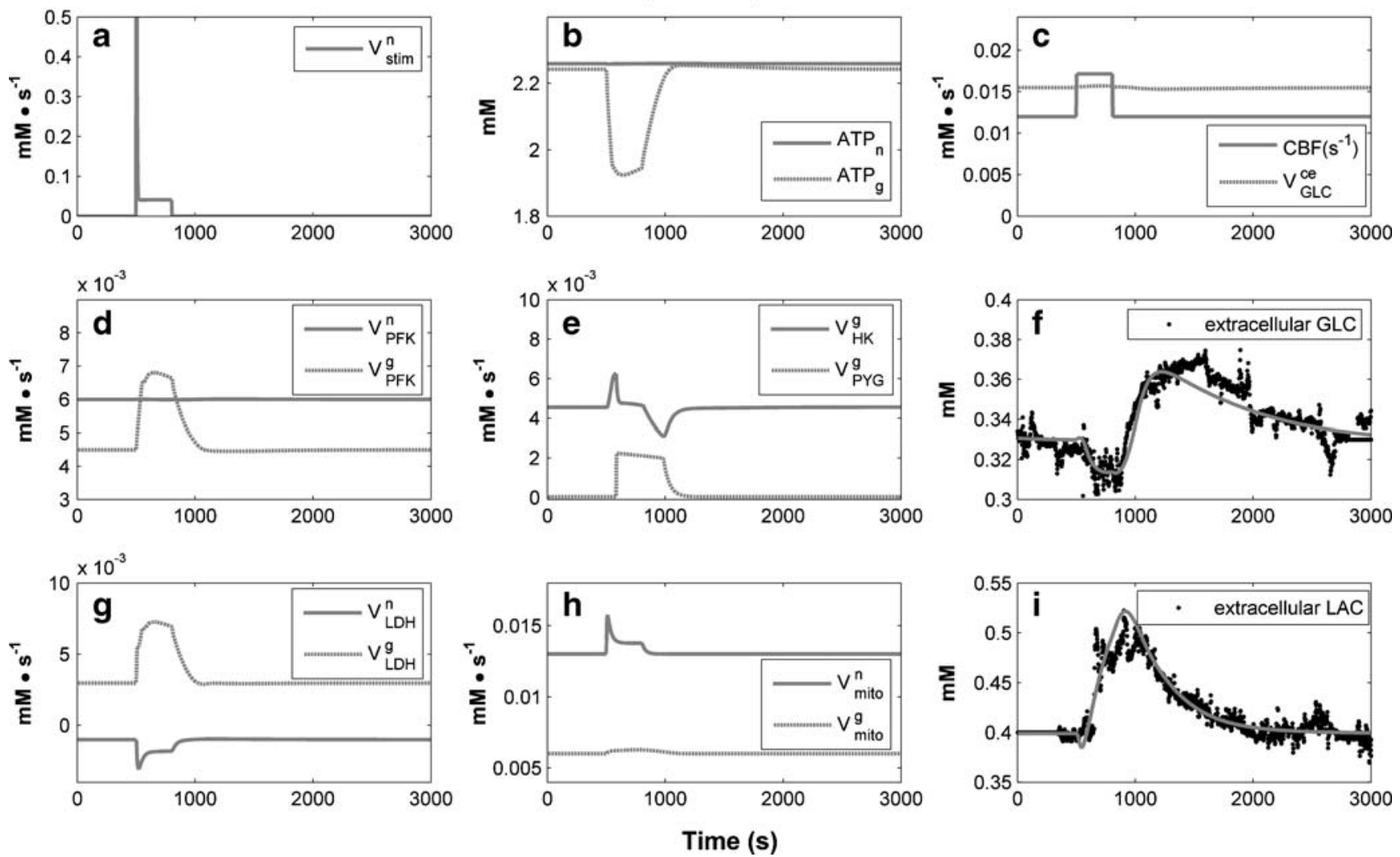

Fig. 2 Results and simulations for a $5 \mathrm{~min}$. tail pinch experiment. GLC and LAC (dots in ' $f$ ' and ' $i$ ') were measured in brain extracellular space using substrate specific microelectrochemical sensors implanted in freely-moving animals and used for model

addition, a 70 seconds delay (post-stimulation) before the increase in GLY breakdown was identified through the calibration routine. The effect of the noradrenaline 'signal' for GLY breakdown is also longer than the stimulation duration $(\approx 400$ s of GLY breakdown for a 300 s stimulation).

The increase in GLY breakdown allows astrocytes to switch from a pure GLC usage to a mix of GLC and GLY usage. The comparison of hexokinase rate $\left(\mathrm{v}_{\mathrm{HK}}{ }^{\mathrm{g}}\right)$ and glycogen breakdown $\left(\mathrm{V}_{\mathrm{PYG}^{\mathrm{g}}}{ }^{\mathrm{g}}\right.$ ) in astrocytes (Fig. 2e) provides a clear explanation for the observed triphasic behaviour for GLC. First, at the onset of stimulation, the activation of glycolysis in astrocytes induces an increase in the $\mathrm{v}_{\mathrm{HK}}{ }^{\mathrm{g}}$ rate (Fig. 2e), this explains why the GLC concentration decreases quickly. Then, after a delay, the noradrenaline 'signal' induces GLY breakdown, which in turn supplies the excess GLC needed in astrocytes. As a result, $\mathrm{v}_{\mathrm{HK}}{ }^{\mathrm{g}}$ goes back to its steady-state value as soon as the GLY breakdown is initiated, and this stabilizes the extracellular GLC concentration. After stimulation ends, the noradrenaline signal is still activating GLY breakdown for $\approx$ $170 \mathrm{~s}$, inducing a consequent increase in G6P (simulation calibration. Stimulation profile $\mathbf{a} ; \mathrm{ATP} \mathbf{b} ; \mathrm{CBF}$ and GLC transport rate c; PFK reaction rate $\mathbf{d}$; HK reaction rate and GLY breakdown in glia $\mathbf{e}$; Extracellular GLC f; LDH reaction rate $\mathbf{g}$; Mitochondrial oxidation rate $\mathbf{h}$; Extracellular LAC i;

results not shown). This in turn inhibits $\mathrm{v}_{\mathrm{HK}}{ }^{\mathrm{g}}$ and results in a fast increase in extracellular GLC.

Interestingly, using the pool of stored GLY during high demand periods allows the cerebral tissue to meet its increased energy requirements with minimal changes in GLC transport from the capillaries (see $\mathrm{v}_{\mathrm{GLC}}{ }^{\mathrm{ce}}$ in Fig. 2c). This allows GLC levels to be maintained in the cerebral environment during stimulation, a factor that is critical for neuronal glycolysis (neurons don't accumulate GLY). The described GLY breakdown $\left(2 \times 10^{-3} \mathrm{mM} \cdot \mathrm{s}^{-1}\right.$ for $\left.400 \mathrm{~s}\right)$ would lead to a dip of $\approx 0.8 \mathrm{mM}$ in astrocytic GLY concentration. This is consistent with the current literature on GLY levels in astrocytes, where Brown and Ransom (2007) reported GLY concentrations in astrocytes in the range of 1.4 to $4.2 \mathrm{mM}$ (adjusted for consistency of units). Simulation results from the model also shows that the GLY pool is replenished in resting conditions, allowing an overall balance between high and low activity periods (simulations not shown). GLY is thus considered here not as an 'infinite' substrate pool, but rather as a dynamic energy reserve that the cerebral tissue can use to buffer its 'energy budget' between low and high demand periods. 
5.3 Probing energy metabolism with propanolol perturbation

As mentioned previously, the complexity of cerebral tissue complicates analysis based on experimental results alone and leaves room for alternative explanations. In the specific case of GLY breakdown, it could be argued that the observed increase in GLC is not coming from the action of astrocytic glycogenolysis, but rather from an increase in GLC transport. To further investigate this point, the GLY breakdown hypothesis was compared to in vivo data from experiments with animals which had pharmacologically treated astrocytes. As observed by Fillenz and Lowry (1998), the dynamic behaviour of GLC concentration during TP is affected by propanolol perfusion in the cerebral tissue. Since propanolol mainly affects astrocytes, it is assumed that the difference will be explained by different astrocytic metabolism (both at rest and during stimulation).

The effect of propanolol on astrocytes was investigated using the model by changing 4 parameters for astrocytic GLC metabolism and transport in a manner that corre- sponded to the physiological effect of propanolol. (i.e. a decrease in metabolic reaction rates). It was found that $\mathrm{a} \approx$ $19 \%$ decrease in maximum reaction rate for GLC transport $\left(\mathrm{V}_{\text {max,GLC }}{ }^{\mathrm{eg}}\right)$ was able to fit the different GLC baseline (0.375 mM instead of $0.34 \mathrm{mM})$. Metabolic regulation and response to stimulation in astrocytes was also affected by propanolol. To represent the fact that astrocytes are less activated during stimulation, glutamate cycling rate was reduced by $10 \%$ in the presence of propanolol. The amplitude of the GLY breakdown during stimulation was found to be $18 \%$ of the value for normal conditions $\left(0.36 \times 10^{-3}\right.$ instead of $\left.2 \times 10^{-3} \mathrm{mM} \cdot \mathrm{s}^{-1}\right)$. The signal for GLY breakdown was also slightly delayed, starting at 135 s poststimulation, instead of $70 \mathrm{~s}$ in normal conditions. With these minimal changes in parameters to represent the physiological effect of propanolol on astrocytes, it was possible to use the same model to describe both data sets (TP response with and without propanolol perfusion).

Results and simulation of a 5 min. TP experiment with propanolol perfusion are presented in Fig. 3. The dynamics of GLC in that case clearly show that the lower GLY breakdown ( $\mathrm{V}_{\mathrm{PYG}}^{\mathrm{g}}$ in Fig. 3e) in the model is coherent with

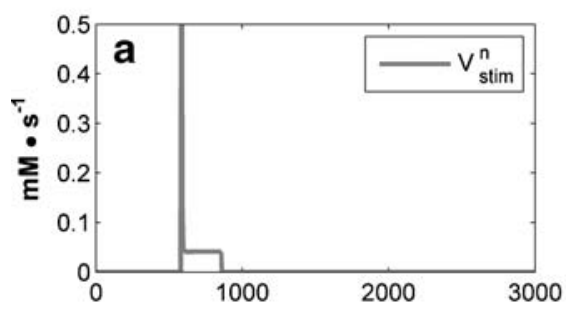

Tail pinch experiment - drugged astrocytes
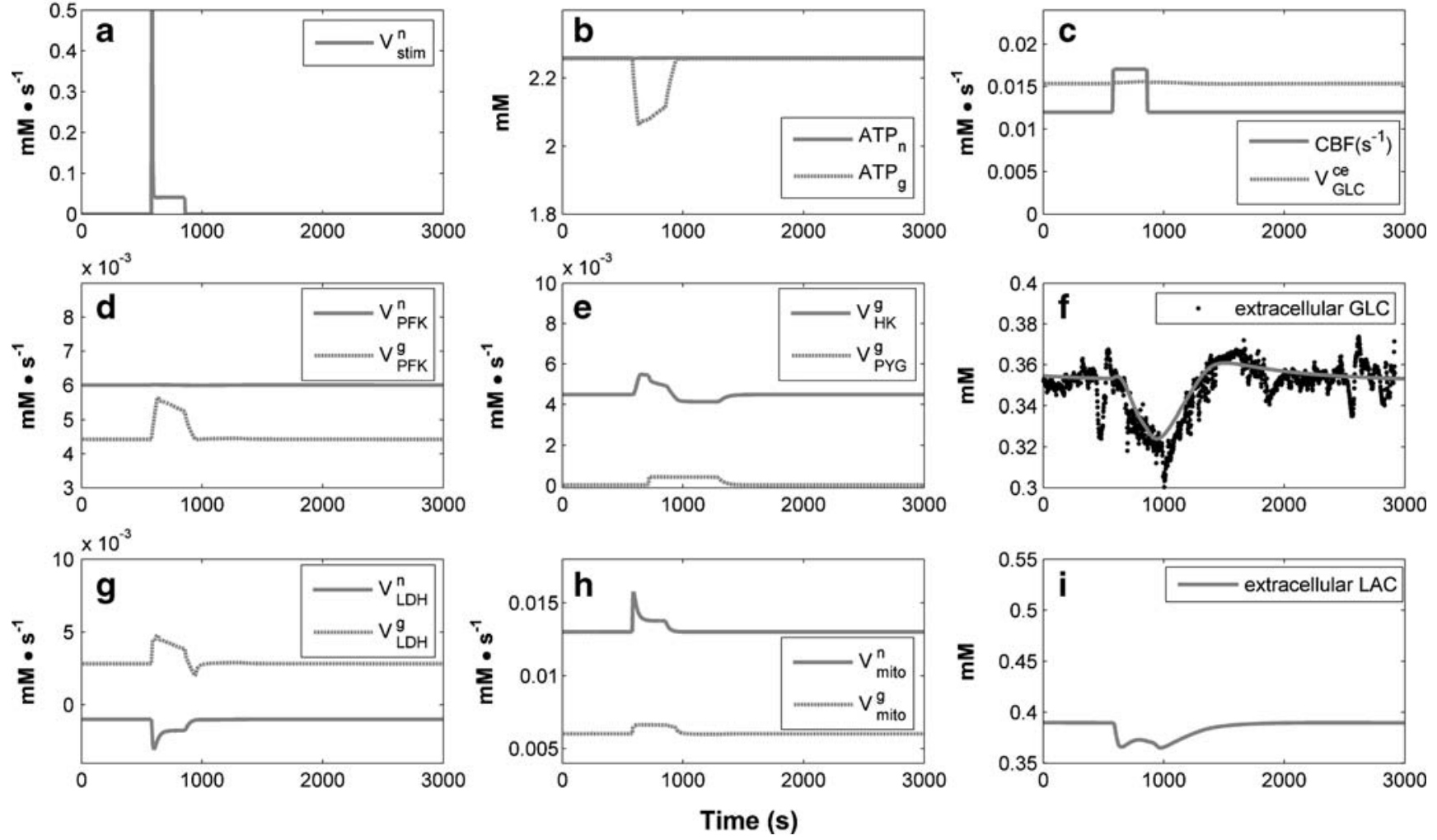

Fig. 3 Results and simulations for a $5 \mathrm{~min}$. tail pinch experiment with propanolol (a beta-adrenoceptor antagonist — astrocytes have a small store of glycogen with a rapid turnover rate, which in cultured astrocytes is broken down by stimulation of beta-adrenoceptors perfusion. GLC was measured in the extracellular space and used for model calibration. Same caption as Fig. 2 applies 
experimental observations. With pharmacologically treated astrocytes, a strict decrease in GLC (during stimulation) followed by recovery is observed (see Fig. 3f). Thus, challenging the dynamic metabolic model with two data sets with different physiological conditions for astrocytes allowed a key mechanism in GLC regulation (secondary activation of GLY breakdown during stimulation) to be identified and quantified. This clarification of the cellular mechanisms for the GLY phenomenon would not have been possible using only steady-state data on normal physiological conditions in the brain.

5.4 Testing the model's predictive capacity with restraint experiments

An important procedure when building a mathematical model for a biological system is its validation. This can be performed by testing the model's capacity to predict results from experimental data sets and inputs (stimuli) that were not used in the calibration routine (Haefner 1996). Thus a different sensory stimulus was used for validation (restraint instead of TP). The restraint stimulus was represented in the model by modifying the input parameters for neuronal stimulation (base stimulation flow, $\mathrm{v}_{\mathrm{n}}{ }^{1}$, early stimulation flow, $\mathrm{v}_{\mathrm{n}}{ }^{2}$ and stimulation time constant, $\mathrm{t}_{\text {stim }}$ ). All remaining parameters of the metabolic model remained unaltered.

Experimental results (in vivo LAC measurements) and the corresponding model simulation of a $5 \mathrm{~min}$. restraint experiment are presented in Fig. 4. The stimulation parameters found by input calibration are $\mathrm{v}_{\mathrm{n}}{ }^{1}=$ $0.019 \mathrm{mM} \cdot \mathrm{s}^{-1}$ (as opposed to 0.041 for TP), $\mathrm{v}_{\mathrm{n}}{ }^{2}=$ $1.6 \mathrm{mM} \cdot \mathrm{s}^{-1}$ (as opposed to 2.56 for TP) and $\mathrm{t}_{\mathrm{stim}}=5.2 \mathrm{~s}$ (as opposed to 2 for TP). Thus, it would seem that the restraint is producing a lower stimulation rate, although the spike at the onset of stimulation is slightly broader (because of a higher $\left.t_{\text {stim }}\right)$. With this modified input, the model was able to correctly predict the measured LAC profile during the restraint experiment. Interestingly, the LAC profile in the restraint case shows a slight dip in the beginning, which is also seen in the experimental data (see Fig. 4i). This initial dip in LAC followed by an overshoot is reported for other types of stimulations (see Aubert and Costalat 2005 and references therein).

From these results, it is asserted that the integrative modelling approach proposed here with neuronal stimulation, GLU cycling and GLY metabolism is appropriate for
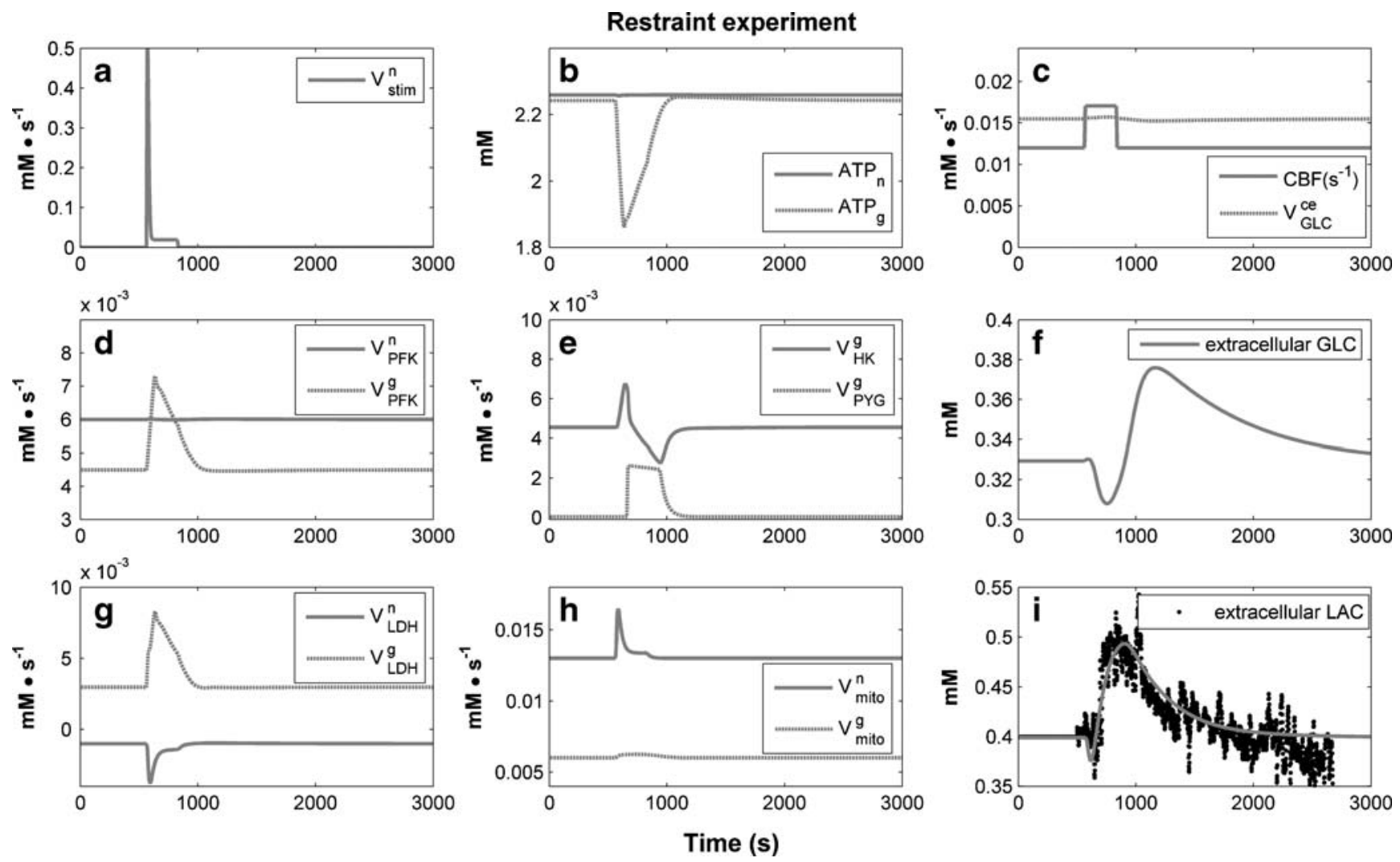

Fig. 4 Results and simulations for a 5 min. restraint experiment. LAC was measured in the extracellular space and used for model calibration. Same caption as Fig. 2 applies 
the description of the overall dynamic coupling of neuronal and astrocytic activity. The fact that a single set of model parameters could describe steady-state behaviour (see Appendix for further calibration with steady-state values) and three different dynamic experiments is an indication of the model's robustness. However, it is clear that many parameters and variables were not substantially analysed in this study and thus, the modelling approach could be further improved by including more in vivo data as it becomes available.

\subsection{Sensitivity analysis}

A sensitivity analysis on the model parameters can be used to obtain further insights into the properties of the metabolic system. This can be performed in many different ways, however, since the dynamic properties of the system seem to be important and seem to be accurately characterised by the model, the variations in parameter sensitivities will be calculated over time. This approach consists of calculating the 'parameter trajectories' (i.e. dynamic change in model behaviour after a parameter change). Defining the change in 'model behaviour' is an important step in this analysis. Here, the weighted sum of metabolites profiles will be used as a criterion to describe 'model behaviour'. Thus, a change in a parameter that affects more metabolite profiles or that has a higher impact on these profiles will be measured as being more sensitive. The parameter sensitivities or parameter trajectories $\left(\mathrm{T}_{\mathrm{j}}\right)$ will thus be described as follows:

$T_{j}(t)=\frac{1}{n} \cdot \frac{\sum_{i}^{n}\left(\left|\Delta S_{i}(t)\right| / S_{i, s s}\right)}{\left|\Delta p_{j}\right| / p_{j}}$

where $S_{i}(t)$ is the concentration profile of the $i^{\text {th }}$ state of the model (here $n=34$ ) and $p_{j}$ is the $j^{\text {th }}$ parameter of the model (here $\mathrm{j}=68$ parameters +26 constants). A variation in $\mathrm{S}_{\mathrm{i}}(\mathrm{t})$ after a parameter change is thus calculated by $\Delta \mathrm{S}_{\mathrm{i}}(\mathrm{t})$. These variations in concentration profiles are weighted (with the steady-state conditions $\mathrm{S}_{\mathrm{i}, \mathrm{ss}}$ ) and summed to calculate the absolute change in 'model behaviour'. This change is then divided by the relative change in parameters (here $\Delta \mathrm{p}_{\mathrm{j}}=1 \%$ of $\mathrm{p}_{\mathrm{j}}$ ). Thus, $\mathrm{T}_{\mathrm{j}}(\mathrm{t})$ is the percent change in model behaviour over time after a $1 \%$ change in parameter value. A value of $\mathrm{T}_{\mathrm{j}}$ of 1 would thus mean that a change of $1 \%$ in parameter would lead to an average change in $1 \%$ for the model states. The results of the parameter trajectories analysis are presented in Fig. 5. For clarity, only the most sensitive parameters are shown $\left(\mathrm{T}_{\mathrm{j}}>0.1\right)$. The results can be separated in sub-categories. First, it was observed that the most sensitive parameters were related either to energy metabolism (Fig. 5a, c) or transport and physiology (Fig. 5b, d). These two categories can also be sub-divided
Fig. 5 Parameter trajectories calculated from the calibrated model. $T_{j}(t)$ is the average percent change over time in model states after a $1 \%$ change in parameter value. Parameters with $\mathrm{T}_{\mathrm{j}}<0.1$ are not shown. Energy related parameters a and c, transport and physiological parameters $\mathbf{b}$ and $\mathbf{d}$

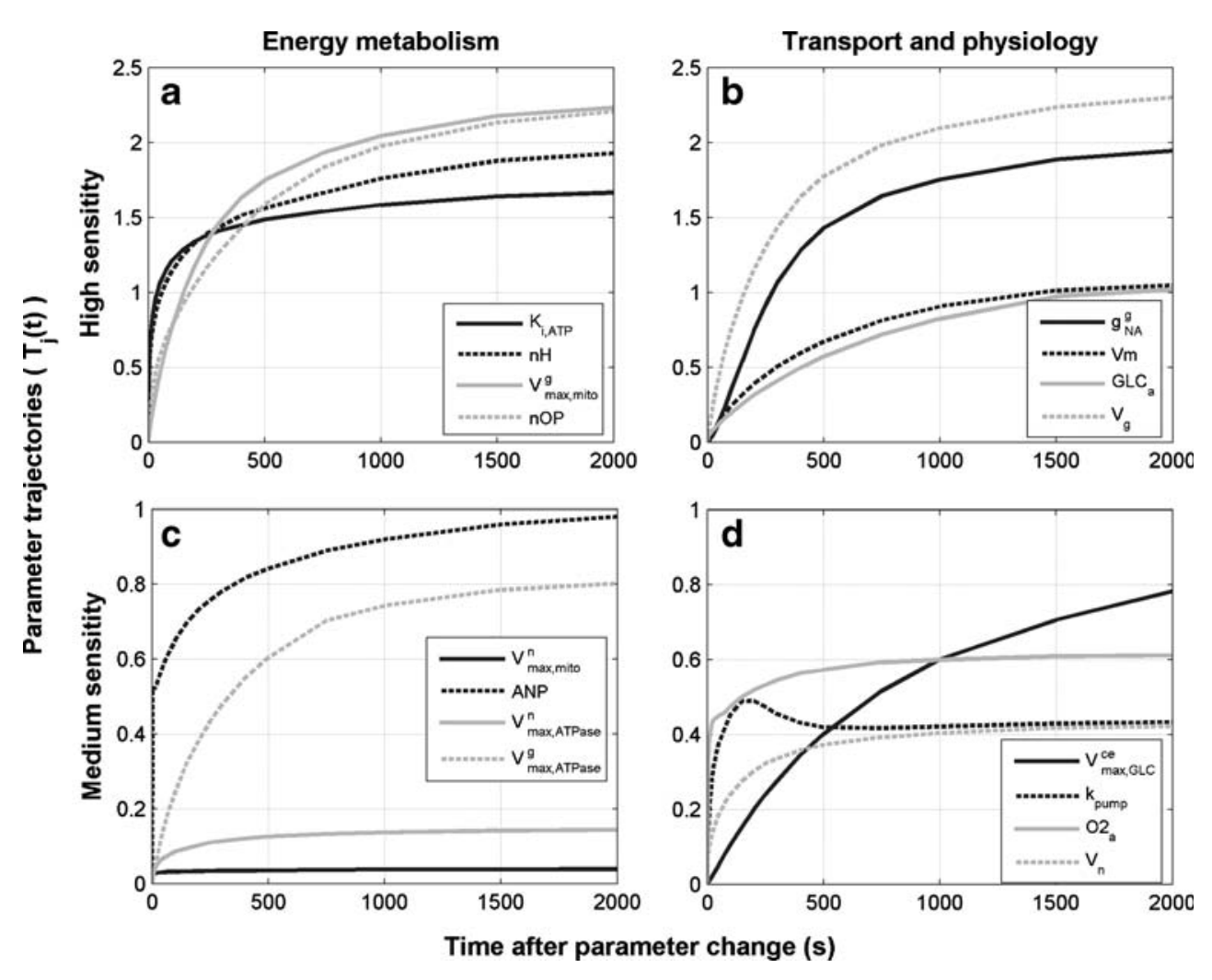


into high-sensitivity parameters $\left(\mathrm{T}_{\mathrm{j}}>1\right.$, Fig. 5a, b) and medium-sensitivity parameters $\left(0.1<\mathrm{T}_{\mathrm{j}}<1\right.$, Fig. $\left.5 \mathrm{c}, \mathrm{d}\right)$.

\subsection{Sensitivity of energy metabolism}

The regulation of energy metabolism seems to be critical for the correct functioning of brain metabolism. The most sensitive parameters in this regard (Fig. 5a) are the mitochondrial maximum reaction rate in astrocytes $\left(\mathrm{V}_{\max }\right.$, mito ${ }^{g}$ ) and the oxidative phosphorylation ratio (nOP, the ratio between moles of ATP produced by moles of PYR oxidized). Thus, the ability of neurons and astrocytes to produce energy significantly affects the overall behaviour of cerebral metabolism. The regulation of the PFK complex by ATP (see Appendix A.1, Table A.1, $v_{\mathrm{PFK}}{ }^{\mathrm{n}}$ ) is considered to be an important control mechanism for glycolysis. Underwood and Newsholme (1965) measured an activity profile of PFK as regards to ATP that showed the activation-inhibition kinetic that is now used extensively (see Heinrich and Schuster 1996; Aubert and Costalat 2005). This regulation is included in the model and is defined by an inhibition constant $\left(\mathrm{K}_{\mathrm{i}, \mathrm{ATP}}\right)$ and an exponent $(\mathrm{nH})$. Interestingly, these two parameters are among the most sensitive parameters in the model (see Fig. 5a). The consumption of ATP by the cells is often mentioned as the driving mechanism for glycolysis (hence the activation of glycolysis during Na-ATPase activation). The sensitivity of the model to the ATPases was indeed observed by the parameter trajectories (Fig. 5c). The fact that both ATPproducing and ATP-consuming processes are sensitive is also in accordance with results by Ainscow and Brand (1999) who studied energetic metabolism control in rat hepatocytes. Finally, the total amount of energetic shuttles (ANP) is also a sensitive parameter in this study.

\subsection{Sensitivity of transport processes}

The regulation of energy metabolism is not the only sensitive process in the model. As shown in Fig. 5b, d, parameters related to nutrient transport $\left(\mathrm{T}_{\max , \mathrm{GLC}}{ }^{\mathrm{ce}}, \mathrm{GLC}_{\mathrm{a}}\right.$, $\mathrm{O}_{2 \mathrm{a}}$ ) induce moderate-to-high changes in model behaviour. Interestingly, the changes in GLC transport parameters take more time to induce changes in model behaviour, whereas a change in arterial $\mathrm{O}_{2}$ induces an almost immediate change in metabolic behaviour. This phenomenon was also observed experimentally by Fillenz and Lowry (1998), where experiments with atmospheric $\mathrm{O}_{2}$ modulation on freely moving rats produced rapid changes in metabolic behaviour. The sensitivity of nutrient transporters (observed experimentally by Simpson et al. 2007) could also represent a physical limitation for the system (i.e. the tissue produces a limited amount of energy for each mole of GLC and $\mathrm{O}_{2}$ available), hence the observed sensitivity. Changes in sodium transport parameters $\left(\mathrm{g}_{\mathrm{NA}}{ }^{\mathrm{g}}, \mathrm{k}_{\text {pump }}, \mathrm{Vm}\right)$ also affect metabolic behaviour. However, this observed sensitivity could be caused by the direct links between sodium transport and energy metabolism. Finally, the relative volumetric fractions of neurons and astrocytes $\left(\mathrm{V}_{\mathrm{n}}\right.$ and $\left.\mathrm{V}_{\mathrm{g}}\right)$ also show moderate $\left(\mathrm{V}_{\mathrm{n}}\right)$ and high $\left(\mathrm{V}_{\mathrm{g}}\right)$ sensitivity.

\subsection{Insensitivity of energy metabolism}

It is interesting to note that many of the kinetic parameters of energy metabolism, namely all the regulation for the reactions involved in glycolysis (except PFK), were among the least sensitive parameters in the model $\left(\mathrm{T}_{\mathrm{j}}<0.1\right)$. This observation suggests that the energy metabolism is a robust energy 'generator' driven mostly by cellular energetic requirements.

\section{Conclusion}

An integrative dynamic modelling approach for brain energy metabolism has been presented. The calibration of model parameters with information from the literature and in vivo GLC and LAC concentration data during $5 \mathrm{~min}$. tail pinch allowed critical improvements on the model's descriptive capacity. A key mechanism in energy metabolism, the activation of GLY breakdown in astrocytes during stimulation, was identified and quantified. Tail pinch experiments with propanolol perfusion allowed further validation of this phenomenon. The model was also able to describe the dynamic response of brain energy metabolism with different stimulation inputs (restraint and TP). The calibrated model was then used to calculate parameter trajectories, a methodology that allowed energy regulation and nutrient transport to be identified as key mechanisms in the metabolic behaviour of the brain. The model resulting from this study is thus generic enough to be further used to integrate (in a systems perspective) available knowledge on brain metabolism. One possibility here would be to analyze different brain regions and different stimuli. Since the model describes the overall metabolic balance and the input stimulation is easily adjustable, it could be adapted to many in vivo or in vitro experimental conditions.

\subsection{Some remarks on ANLS}

Simulation results suggest that the observed dynamics for GLC and LAC during stimulation support the ANLS hypothesis. For example, the lactate dehydrogenase (LDH) simulated fluxes in neurons and astrocytes shows a shuttling (see for example Fig. $2 \mathrm{~g}: v_{\mathrm{LDH}}{ }^{\mathrm{g}}>0$ while $v_{\mathrm{LDH}}{ }^{\mathrm{n}}<$ $0)$. The associated transport rate $\left(\nu_{\mathrm{LAC}}{ }^{\mathrm{ge}}>0\right.$ and $\left.\nu_{\mathrm{LAC}}{ }^{\mathrm{ne}}<0\right)$ simulations (not shown) are also coherent with a shuttling. 
The parameter calibration routine with experimental data thus led to a model that exhibits ANLS behaviour, although, as in Aubert and Costalat (2005), no special features of the model are especially favourable to the ANLS hypothesis. However, it must be noted that the model is far from a 'full' LAC shuttling, as glycolysis is still providing neurons with PYR, and the LAC produced by astrocytes is partially cleared by the capillary. In resting conditions, only $7 \%$ of the PYR consumed by neuronal mitochondria comes from LAC, a proportion that rises to $12-15 \%$ during stimulation. This allowed neurons to perform their 'stimulatory work' with minimal changes in ATP, as the LAC inflow in neurons is quickly channelled to mitochondria (Fig. $2 \mathrm{~h}, v_{\text {mito }}{ }^{\mathrm{n}}$ ). Simulations also show that LAC shuttling will depend on the intensity of stimulation and thus, different rates of ANLS could be observed, as was the case in works by Aubert and co-workers. However, further analysis will be required to clarify this point, as the analytical and experimental technologies are now sufficiently mature to quantitatively assess these biological hypotheses in an integrative perspective. For example, experimental $\mathrm{O}_{2}$ data was not used in this study, but the modelling approach could be used to analyze such data and maybe provide further insight on the dynamics and compartmentalisation of oxidative vs. non-oxidative metabolism in the brain. In this context, the precise description of metabolic mechanisms by using dynamic models calibrated with quantitative data will be the key to provide answers.

\subsection{Supplemental information}

The model presented in this study is available on the Biomodels database (www.ebi.ac.uk/biomodels-main) in SBML format.

Acknowledgements The authors wish to thank Marianne Fillenz for suggesting critical improvements to the model, especially regarding glycogen storage and utilization in astrocytes.

\section{Appendix A: Mass balances and kinetic equations of the brain energy metabolism model}

This Appendix presents the mass balances, kinetic equations and parameters of the model.

The metabolic model is presented as a group of interacting subsystems that describe specific cellular functions, while performing critical interactions with other subsystems. It is our belief that the decomposition of the model will allow a better systems understanding of the problem of simulating brain physiology. The subsystems considered in the modelling are described in the following Appendixes:

(A1) Compartments and exchange systems (GLC, LAC, $\mathrm{O} 2, \mathrm{CO} 2)$

(A2) Central energy metabolism (glycolysis + mitochondrial oxidation)

(A3) Neuronal stimulation system and glutamate cycling for astrocytes-neurons coordination

(A4) Glycogen storage (astrocytes only)

Each of these cellular subsystems will be described in detail (differential equations and kinetic parameters). Appendix A.5 then presents an overview of the complete model. A complete set of model parameters is presented in section A.6. Regulation mechanisms and changes in behaviour in the model were described using a 'switch function' $(\mathrm{f}(\mathrm{t}, \delta, \mathrm{a}))$ which is described in section A.7.

\section{A.1 Compartments and exchange systems}

The exchange systems account for the transfer fluxes between the cerebral compartments Fig. 6. The model considers 4 compartments:

- Capillary $\left(\mathrm{V}_{\mathrm{c}}\right)=0.55 \%$ of total volume

- Extracellular space $\left(\mathrm{V}_{\mathrm{e}}\right)=20 \%$ of total volume

- Neuronal volume $\left(\mathrm{V}_{\mathrm{n}}\right)=45 \%$ of total volume

- Astrocytic, or glial volume $\left(\mathrm{V}_{\mathrm{g}}\right)=25 \%$ of total volume

Fig. 6 Exchange systems

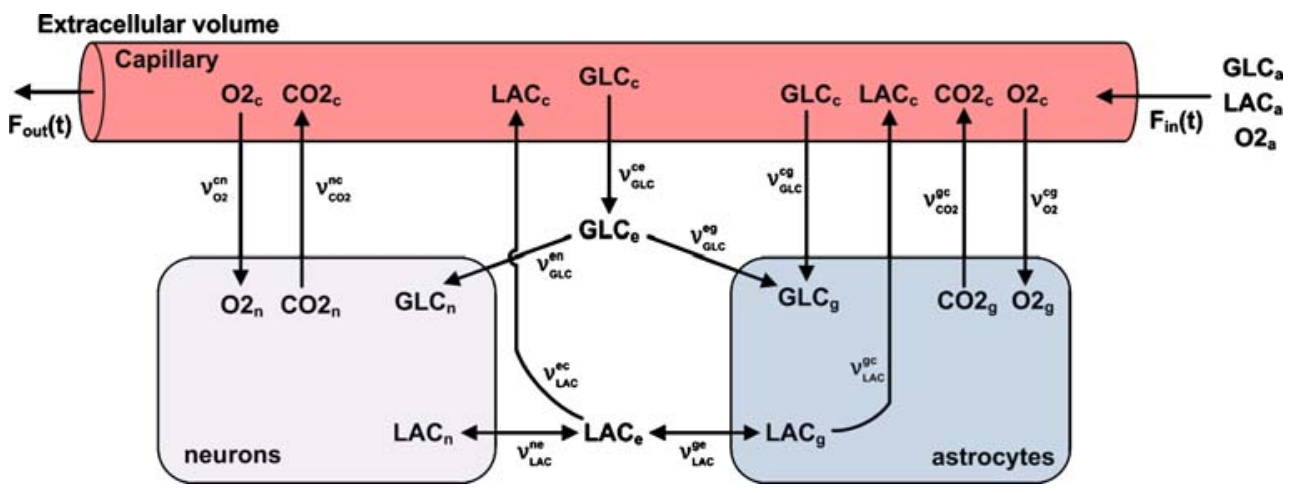


The exchange fluxes between these compartments consider the compartment's volumetric ratios in order to have consistent mass balances (for example $\mathrm{R}_{\mathrm{cg}}=\mathrm{V}_{\mathrm{c}} / \mathrm{V}_{\mathrm{g}}=$ $0.055 / 0.25$ for exchanges between capillaries and astrocytes). Each exchange flux is defined with a given compartment as reference volume, so no volumetric adjustment is required in these specific cases. The following hypotheses are made to describe the transfer of substrate and by-products of cerebral metabolism:

- Transfer of gaseous species (O2 and $\mathrm{CO} 2)$ between cellular compartments and capillaries is assumed to be direct. $\mathrm{O} 2$ transfer is described with the mechanisms used in Aubert and Costalat (2005).

- GLC and LAC exchanges between the compartments are regulated by transporters that are described by facilitated diffusion equations.

- CBF regulation was built upon works by Aubert and Costalat (2005), but the regulation of blood flow in this study is described using a generic sigmoid switch (see Appendix A.6).

- No hypotheses are made as regards the ANLS. Lactate transfer is described by facilitated diffusion and transfer from astrocytes to neurons occurs only if the gradients are favourable. Since two mechanisms excrete lactate from the cerebral tissue ( $\mathrm{v}^{\mathrm{ec}}$ LAC and $\mathrm{v}^{\mathrm{gc}}$ LAC $)$, there is no 'structural bias' in the model to force lactate transfer from astrocytes to neurons Tables 1 and 2.

\section{A.2 Central energy metabolism}

Figure A-2 presents the reactions that are considered for central energy metabolism. In this work, we consider the processing of GLC through glycolysis, with mitochondrial oxidation of PYR to be the major pathways of central energy metabolism. The action of ATPases and phosphocreatine (PCr) buffering, as well as the action of lactate dehydrogenase $(\mathrm{vLDH})$ are considered. These reactions occur in both neurons and astrocytes Fig. 7.

- Glycolysis is simplified and represented by 5 reactions but these still accounts for ATP/ADP and NAD/NADH balances and regulation (discussed in the text).

- Glycolytic reactions include the same reactions as were presented in works by Aubert and Costalat (2005), with the difference that the conversion of GLC to GAP is now described in three reactions instead of one. This allows a better description of regulatory phenomenon, especially as regards to glycogen storage (astrocytes, see Appendix A.4), and GLC consumption dynamics by the hexokinase (vHK) a reaction highly inhibited by its product, G6P.

- A constant amount of energetic shuttles (ATP+ADP+ AMP $=$ ANP) is assumed. Distribution between ATP, $\mathrm{ADP}$ and AMP varies depending on energetic requirements, kinetic reactions and adenylate kinase equilibrium. Equilibrium description from Heinrich and Schuster (1996) was used for adenylate kinase.

Table 1 Variables for exchange and transport systems

\begin{tabular}{|c|c|c|c|c|}
\hline Variable & & Steady-state value & Differential equation & \\
\hline \multicolumn{5}{|l|}{ Extracellular states } \\
\hline GLCe & Glucose & 0.47 & \multirow{2}{*}{\multicolumn{2}{|c|}{$\begin{array}{l}\frac{\partial G L C_{e}}{\partial t}=v_{G L C}^{c e}-R_{n e} \cdot v_{G L C}^{e n}-R_{g e} \cdot v_{G L C}^{c e} \\
\frac{\partial L A C_{e}}{\partial t}=\mathrm{R}_{\mathrm{en}} \cdot v_{\mathrm{LAC}}^{\mathrm{ne}}+\mathrm{R}_{\mathrm{eg}} \cdot v_{\mathrm{LAC}}^{\mathrm{ge}}-v_{\mathrm{LAC}}^{\mathrm{ec}}\end{array}$}} \\
\hline $\mathrm{LACe}$ & Lactate & 0.37 & & \\
\hline \multicolumn{5}{|l|}{ Astrocytic and neuronal states } \\
\hline $\mathrm{O} 2 \mathrm{n}$ & Oxygen & 0.102 & \multirow{2}{*}{\multicolumn{2}{|c|}{$\begin{array}{l}\frac{\partial O 2_{n}}{\partial t}=v_{O 2}^{c n}-3 \cdot v_{m i t o}^{n} \\
\frac{\partial O 2_{g}}{\partial t}=v_{O 2}^{c g}-3 \cdot v_{m i t o}^{g}\end{array}$}} \\
\hline $\mathrm{O} 2 \mathrm{~g}$ & Oxygen & 0.102 & & \\
\hline \multicolumn{5}{|l|}{ Capillary } \\
\hline $\mathrm{O} 2 \mathrm{c}$ & Oxygen & 7.46 & \multirow{4}{*}{\multicolumn{2}{|c|}{$\begin{array}{l}\frac{\partial O 2_{c}}{\partial t}=v_{O 2}^{c}-R_{c g} v_{O 2}^{c g}+R_{c n} \cdot v_{O 2}^{c n} \\
\frac{\partial C O 2_{c}}{\partial t}=v_{C O 2}^{c}-R_{c g} \cdot v_{C O 2}^{c g}+R_{c n} \cdot v_{l C O 2}^{c n} \\
\frac{\partial G L C_{c}}{\partial t}=v_{G L C}^{c}-\mathrm{R}_{\mathrm{ec}} \cdot v_{\mathrm{GLC}}^{\mathrm{ce}}-\mathrm{R}_{\mathrm{gc}} \cdot v_{\mathrm{GLC}}^{\mathrm{cg}} \\
\frac{\partial L A C_{c}}{\partial t}=v_{L A C}^{c}-\mathrm{R}_{\mathrm{ec}} \cdot v_{\mathrm{GLC}}^{\mathrm{ce}}-\mathrm{R}_{\mathrm{gc}} \cdot v_{\mathrm{LAC}}^{\mathrm{gc}}\end{array}$}} \\
\hline $\mathrm{CO} 2 \mathrm{c}$ & Carbon dioxide & 0.98 & & \\
\hline GLCc & Glucose & 4.64 & & \\
\hline LACc & Lactate & 0.33 & & \\
\hline \multicolumn{5}{|l|}{ Other states and variables } \\
\hline $\mathrm{Vv}$ & Venous volume & 0.0237 & $\frac{\partial V_{v}}{\partial t}=F_{\text {in }}(t)-F_{\text {out }}(t)$ & \\
\hline $\mathrm{F}_{\text {in }}$ & Capillary entering & 0.012 & $F_{i n}(t)=F_{o}+f_{C B F}(t)$ & \\
\hline \multirow[t]{2}{*}{$\mathrm{F}_{\text {out }}$ Flow out of the capillary $\left(\mathrm{s}^{-1}\right)$} & $\begin{array}{l}\text { blood flow }\left(\mathrm{s}^{-1}\right) \\
0.012\end{array}$ & & \multirow{2}{*}{\multicolumn{2}{|c|}{$F_{\text {out }}(t)=F_{0} \cdot\left[\left(\frac{V v}{V v, 0}\right)^{1 / x}+\tau_{v}\left(\frac{V v}{V v, 0}\right)^{-1 / 2} \cdot \frac{1}{V v, 0} \frac{d V}{d t}\right]$}} \\
\hline & $\mathrm{CBF}$ regulation & & & \\
\hline
\end{tabular}

$*_{\mathrm{t}}$ is the time post-stimulation, tend is the duration of stimulation. A delay of $2 \mathrm{sec}$. (poststimulation) affects the variations in CBF. The function ' $\mathrm{f}$ $(\mathrm{t}, \delta, \alpha)^{\prime}$ used to build $\mathrm{fCBF}(\mathrm{t})$ is described in Appendix A.7 
Table 2 Kinetic equations for exchange and transport systems

\begin{tabular}{|c|c|c|}
\hline No. & Reaction & Kinetic equation \\
\hline \multicolumn{3}{|c|}{ Transport } \\
\hline 1 & GLC exchange between capillary and extracellular fluid & $v_{G L C}^{c e}=V_{\max , G L C}^{c e} \cdot\left[\frac{G L C_{c}}{G L C_{c}+K_{T, G L C}^{c e}}-\frac{G L C_{e}}{G L C_{e}+K_{T, G L C}^{c e}}\right.$ \\
\hline 2 & GLC exchange between capillary and astrocytes & $v_{G L C}^{c g}=V_{\max , G L C}^{e n} \cdot\left[\frac{G L C_{c}}{G L C_{c}+K_{T, G L C}^{c g}}-\frac{G L C_{g}}{G L C_{g}+K_{T, G L C}^{c g}}\right.$ \\
\hline 3 & GLC exchange between extracellular fluid and neurons & $v_{G L C}^{e n}=V_{\max , G L C}^{c g} \cdot\left[\frac{G L C_{e}}{G L C_{c}+K_{T, G L C}^{e n}}-\frac{G L C_{n}}{G L C_{n}+K_{T, G L C}^{e n}}\right.$ \\
\hline 4 & GLC exchange between extracellular fluid and astrocytes & $v_{G L C}^{e g}=V_{\max , G L C}^{e g} \cdot\left[\frac{G L C_{e}}{G L C_{e}+K_{T, G L C}^{e g}}-\frac{G L C_{g}}{G L C_{g}+K_{T, G L C}^{e g}}\right.$ \\
\hline 5 & LAC exchange between extracellular fluid and capillary & $v_{L A C}^{e c}=V_{\max , L A C}^{e c} \cdot\left[\frac{L A C_{e}}{L A C_{e}+K_{T, L A C}^{e c}}-\frac{G L C_{c}}{G L C_{c}+K_{T, L A C}^{e c}}\right]$ \\
\hline 6 & LAC exchange between neurons and extracellular fluid & $v_{L A C}^{n e}=V_{\max , L A C}^{n e} \cdot\left[\frac{L A C_{n}}{L A C_{n}+K_{T, L A C}^{n e}}-\frac{L A C_{e}}{G L C_{e}+K_{T, L A C}^{n e}}\right]$ \\
\hline 7 & LAC exchange between astrocytes and extracellular fluid & $v_{L A C}^{g e}=V_{\max , L A C}^{g e} \cdot\left[\frac{L A C_{g}}{L A C_{g}+K_{T, L A C}^{g e}}-\frac{L A C_{e}}{G L C_{e}+K_{T, L A C}^{g e}}\right.$ \\
\hline 8 & LAC exchange between astrocytes and capillary & $v_{L A C}^{g c}=V_{\max , L A C}^{g c} \cdot\left[\frac{L A C_{g}}{L A C_{g}+K_{T, L A C}^{g c}}-\frac{L A C_{c}}{G L C_{e}+K_{T, L A C}^{g c}}\right.$ \\
\hline 9 & $\mathrm{O} 2$ exchange between capillary and neurons & $v_{O 2}^{c n}=\frac{P S_{c a p}}{V_{n}} \cdot\left[K_{02}\left(\frac{H b . O P}{O 2_{c}}-1\right)^{-1 / n h}-O 2_{n}\right]$ \\
\hline 10 & $\mathrm{O} 2$ exchange between capillary and astrocytes & $v_{O 2}^{c g}=\frac{P S_{c a p}}{V_{g}} \cdot\left[K_{02}\left(\frac{H b . O P}{O 2_{c}}-1\right)^{-1 / n h}-O 2_{g}\right]$ \\
\hline 11 & Blood flow contribution to capillary $\mathrm{O} 2$ & $v_{O 2}^{c a p}=\frac{2 \cdot F_{i n}(t)}{V_{c}} \cdot\left(O 2_{a}-O 2_{c}\right)$ \\
\hline 12 & Blood flow contribution to capillary GLC & $v_{G L C}^{c a p}=\frac{2 \cdot F_{i n}(t)}{V_{c}} \cdot\left(G L C_{a}-G L C_{c}\right)$ \\
\hline 13 & Blood flow contribution to capillary LAC & $v_{L A C}^{c a p}=\frac{2 \cdot F_{i n}(t)}{V_{c}} \cdot\left(L A C_{a}-L A C_{c}\right)$ \\
\hline
\end{tabular}

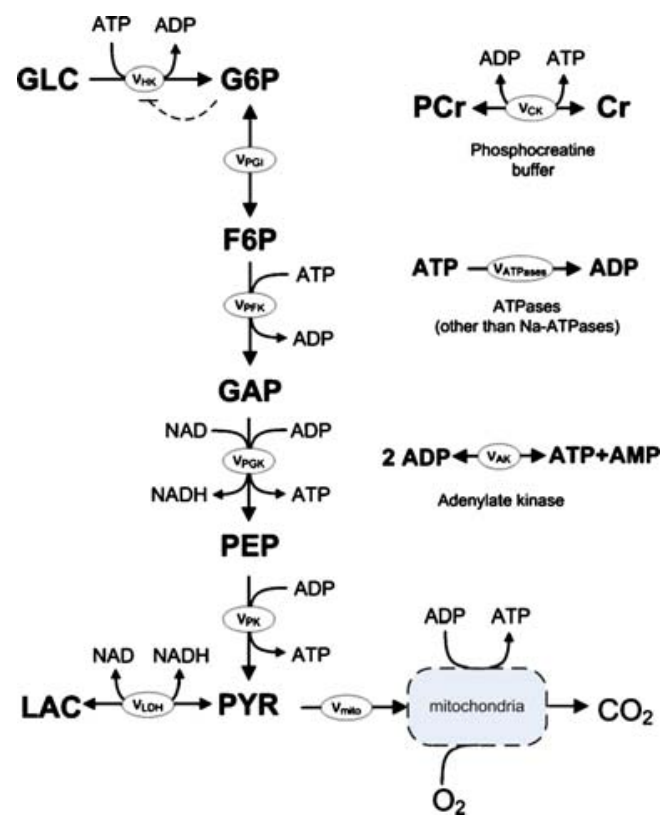

Fig. 7 Central energy metabolism
- The same hypothesis of a constant sum is made for cofactors NADH $+\mathrm{NAD}=$ NADHtot as well as for phosphocreatine buffer $(\mathrm{PCr}+\mathrm{Cr}=\mathrm{PCrtot})$.

- Inhibition of mitochondrial activity at high ATP/ADP ratio is considered (see Table A.2.2, equations 6 and $15)$.

- The stoichiometry and overall mass balances of the reactions of central energy metabolism are the same in neurons and astrocytes, with the same regulation mechanisms. However, the kinetic parameters are different, which allows describing the different metabolic behaviour of neurons and astrocytes at rest or during active periods.

- No hypotheses or fluxes are imposed a priori as regards to the ANLS. The model simply describes the kinetic behaviour of key enzymatic reactions. The Lactate dehydrogenase enzyme, which catalyses the conversion between PYR and LAC, is usually assumed to favour the forward reaction (PYR $\rightarrow$ LAC). This is seen in the parameters (see Table A.6) as the forward rate constant is much higher than the reverse rate constant $\left(\mathrm{k}^{\mathrm{n}}{ }_{\mathrm{LDH}, \mathrm{f}}>\right.$ $\mathrm{k}^{\mathrm{n}}$ LDH,r). However, if the conditions are favourable (NADH/NAD and PYR/LAC ratios), the reaction could produce PYR from LAC Tables 3 and 4. 
Table 3 Central energy metabolism

\begin{tabular}{|c|c|c|c|}
\hline \multicolumn{2}{|l|}{ Variable } & $\begin{array}{l}\text { Steady- } \\
\text { state }\end{array}$ & Differential equation \\
\hline \multicolumn{4}{|c|}{ Neuronal states } \\
\hline GLCn & Glucose & 0.43 & $\frac{\partial G C L_{n}}{\partial t}=v_{G L C}^{e n}-v_{H K}^{n}$ \\
\hline G6Pn & Glucose-6-P & 0.75 & $\frac{\partial G 6 P_{n}}{\partial t}=v_{H K}^{n}-v_{P G 1}^{n}-v_{G 6 P D H}^{n}$ \\
\hline F6Pn & Fructose-6-P & 0.2 & $\frac{\partial F 6 P_{n}}{\partial t}=v_{P G 1}^{n}-v_{P F K}^{n}-v_{P P P}^{n}$ \\
\hline GAPn & Glyceraldehyde-3-P & 0.05 & $\frac{\partial G A P_{n}}{\partial t}=2 \cdot v_{P F K}^{n}-v_{P G K}^{n}-v_{P P P}^{n}$ \\
\hline PEPn & Phosphoenolpyruvate & 0.025 & $\frac{\partial P E P_{n}}{\partial t}=v_{P G K}^{n}-v_{P K}^{n}$ \\
\hline PYRn & Pyruvate & 0.12 & $\frac{\partial P Y R_{n}}{\partial t}=v_{P K}^{n}-v_{L D H}^{n}-v_{\text {mito }}^{n}$ \\
\hline $\mathrm{LACn}$ & Lactate & 0.28 & $\frac{\partial L A C_{n}}{\partial t}=v_{L D H}^{n}-v_{L A C}^{e n}$ \\
\hline ATPn & $\begin{array}{l}\text { Adenosine } \\
\text { triphosphate }\end{array}$ & 2.25 & $\begin{array}{l}\frac{\partial A T P_{n}}{\partial t}=\left[-v_{H K}^{n}-v_{P F K}^{n}+v_{P G K}^{n}+v_{P K}^{n}+15 \cdot v_{\text {mito }}^{n}+v_{C K}^{n}-v_{\text {pump }, N a}^{n}-v_{A T P a s e}^{n}\right] \cdot\left[1-\frac{d A M P_{n}}{d A T P_{n}}\right]^{-1} \\
\text { with } \operatorname{ATP}_{\mathrm{n}}+\mathrm{ADP}_{\mathrm{n}}+\mathrm{AMP}_{\mathrm{n}}=\mathrm{ANP}\end{array}$ \\
\hline NADHn & $\begin{array}{l}\text { Nicotinamide adenine } \\
\text { dinucleotide reduced }\end{array}$ & 0.04 & $\frac{\partial N A D H_{n}}{\partial t}=v_{P G K}^{n}-v_{L D H}^{n}-v_{m i t o}^{n}$ with $\mathrm{NADH}_{\mathrm{n}}+\mathrm{NAD}_{\mathrm{n}}=\mathrm{NADH}_{\text {tot }}$ \\
\hline PCrn & Phosphocreatine & 2.5 & $\frac{\partial P C r_{n}}{\partial t}=-v_{C K}^{n}$ with $\mathrm{PCr}_{\mathrm{n}}+\mathrm{Cr}_{\mathrm{n}}=\mathrm{PCr}_{\text {tot }}$ \\
\hline \multicolumn{4}{|c|}{ Astrocytic states } \\
\hline GLCg & Glucose & 0.16 & $\frac{\partial G L C_{g}}{\partial t}=v_{G L C}^{e g}+v_{G L C}^{c g}-v_{H K}^{g}$ \\
\hline G6Pg & Glucose-6-P & 0.75 & $\frac{\partial G 6 P_{g}}{\partial t}=v_{H K}^{g}+v_{P G 1}^{g}-v_{G L Y S}^{g}+v_{G L Y P}^{g}$ \\
\hline F6Pg & Fructose-6-P & 0.2 & $\frac{\partial F 6 P_{g}}{\partial t}=v_{P G 1}^{g}-v_{P F K}^{g}$ \\
\hline GAPg & Glyceraldehyde-3-P & 0.05 & $\frac{\partial G A P_{g}}{\partial t}=2 \cdot v_{P F K}^{g}-v_{P G K}^{g}$ \\
\hline PEPg & Phosphoenolpyruvate & 0.025 & $\frac{\partial P E P_{g}}{\partial t}=v_{P G K}^{g}-v_{P K}^{g}$ \\
\hline PYRg & Pyruvate & 0.12 & $\frac{\partial P Y R_{g}}{\partial t}=v_{P K}^{g}+v_{L D H}^{g}-v_{m i t o}^{g}$ \\
\hline $\mathrm{LACg}$ & Lactate & 0.89 & $\frac{\partial L A C_{g}}{\partial t}=v_{L D H}^{g}+v_{L A C}^{e g}-v_{L A C}^{g c}$ \\
\hline ATPg & $\begin{array}{l}\text { Adenosine } \\
\text { triphosphate }\end{array}$ & 2.2 & $\begin{array}{l}\frac{\partial A T P_{g}}{\partial t}=\left[-v_{H K}^{g}-v_{P F K}^{g}+v_{P G K}^{g}+v_{P K}^{g}+15 \cdot v_{\text {mito }}^{g}+v_{C K}^{g}-v_{p u m p, N a}^{g}-v_{A T P a s e}^{g}\right] \cdot\left[1-\frac{d A M P_{g}}{d A T P_{g}}\right]^{-1} \\
\text { with ATPn }+\mathrm{ADPn}+\mathrm{AMPn}=\mathrm{ANP}\end{array}$ \\
\hline $\mathrm{NADHg}$ & $\begin{array}{l}\text { Nicotinamide adenine } \\
\text { dinucleotide reduced }\end{array}$ & 0.04 & $\frac{\partial N A D H_{g}}{\partial t}=v_{P G K}^{g}+v_{L D H}^{g}-v_{m i t o}^{g}$ with $\mathrm{NADH}^{\mathrm{g}}+\mathrm{NAD}^{\mathrm{g}}=\mathrm{NADH}^{\text {tot }}$ \\
\hline PCrg & Phosphocreatine & 1.5 & $\frac{\partial P C r_{g}}{\partial t}=-v_{C K}^{g}$ with $\mathrm{PCr}_{\mathrm{g}}+\mathrm{Cr}_{\mathrm{g}}=\mathrm{PCr}_{\text {tot }}$ \\
\hline
\end{tabular}

A.3 Neuronal stimulation and glutamate cycling for astrocytes-neurons coordination

The stimulatory subsystem is built upon works by Aubert and Costalat (2005) to describe neuronal stimulation (vn stim). A critical addition to the stimulation system is the glutamate cycling proposed by Pellerin and Magistretti (1994) for neuronal-astrocytic coordination Fig. 8. The following mechanisms and hypotheses are modelled:

- A sodium inflow ( $\mathrm{v}^{\mathrm{n}}$ stim described by $\mathrm{v}^{\mathrm{n}} 1$ and $\mathrm{v}^{\mathrm{n}} 2$ in Table A.3.2) stimulates neuronal ATPase. This inflow is considered to be an 'input' in the sense that it can be modulated to get a better description of physiological stimulations (strength and duration).

- Upon neuronal stimulation, GLU is released by neurons and taken up by astrocytes (veg

- GLU) with the co-transport of 3 sodium ions.

- The conversion of GLU to glutamine (GLN) in astrocytes for its 'non-stimulatory' transfer to neurons is modelled as one reaction (vgn GLU).

- This simplified model (neglecting GLN) retains the critical feature of the GLU cycling: a coordinated activation of ATPases in astrocytes upon neuronal activation.

- The stoichiometry for ATP consumption in the cycle is respected, as 2 molecules of ATP are required in astrocytes for each molecule of GLU circulating in the cycle (one for Na pumping and one for GLU conversion and transfer).

Considering the dynamics of GLU cycling allows describing cerebral activity with a physiological approach, instead of using two distinct stimulations for neurons and astrocytes, as was the case in Aubert and Costalat (2005). The equations for the stimulation system are presented in Tables 4 and 5 .

\section{A.4 Glycogen storage system}

Figure A-4 presents the mechanisms considered for glycogen storage in astrocytes and its link to central energy metabolism through G6P. The reactions for GLY storage are considered only for astrocytes, as neurons don't accumulate GLY. Glycogen levels in astrocytes can vary 
Table 4 Kinetic equations for central energy metabolism

\begin{tabular}{|c|c|c|}
\hline No. & Reaction & Kinetic equation \\
\hline \multicolumn{3}{|c|}{ Neuronal metabolism } \\
\hline 1 & Hexokinase & $v_{H K}^{n}=k_{H K}^{n} \cdot A T P_{n} \cdot\left[\frac{G L C_{n}}{G L C_{n}+K_{m, G L C}}\right] \cdot[1-f(G 6 P n, 0.6,20)]$ \\
\hline 2 & Phosphoglucose isomerase & $v_{P G 1}^{n}=v_{\max , f, P G 1}^{n} \cdot\left[\frac{G 6 P_{n}}{G 6 P_{n}+K_{m, G G P}}\right]-v_{\max , r, P G 1}^{n} \cdot\left[\frac{F 6 P_{n}}{F 6 P_{n}+K_{m, F G P}}\right]$ \\
\hline 3 & Phosphofructokinase & $v_{P F K}^{n}=k_{P F K}^{n} \cdot A T P_{n} \cdot\left[1+\left(\frac{A T P_{n}}{K_{1, A T P}}\right)^{n H}\right]^{-1} \cdot\left[\frac{F G P_{n}}{F 6 P_{n}+K_{m, F G P}}\right]$ \\
\hline 4 & Phosphoglycerate kinase & $v_{P G K}^{n}=k_{P G K}^{n} \cdot G A P_{n} \cdot A D P_{n} \cdot\left[\frac{N A D_{n}}{N A D H_{n}}\right]$ \\
\hline 5 & Pyruvate kinase & $v_{P K}^{n}=k_{P K}^{n} \cdot P E P_{n} \cdot A D P_{n}$ \\
\hline 6 & Mitochondrial oxidation of pyruvate & $v_{\text {mito }}^{n}=v_{\text {max }, \text { mito }}^{n} \cdot\left[\frac{P Y R_{n}}{P Y R_{n}+K_{m, P Y R}}\right] \cdot\left[\frac{A D P_{n}}{A D P_{n}+K_{m, A D P}}\right] \cdot\left[\frac{O 2_{n}}{O 2_{n}+K_{m, O 2}}\right] \cdot\left[1-f\left(\frac{A T P_{n}}{A D P_{n}}, 20,5\right)\right]$ \\
\hline 7 & Lactate dehydrogenase & $v_{L D H}^{n}=k_{L D H, f}^{n} \cdot P Y R_{n} \cdot N A D H_{n}-k_{L D H, r}^{n} \cdot L A C_{n} \cdot N A D_{n}$ \\
\hline 8 & Creatine kinase & $v_{C K}^{n}=k_{C K, f}^{n} \cdot P C r_{n} \cdot A D P_{n}-k_{C K, r}^{n} \cdot C r_{n} A T P_{n}$ \\
\hline 9 & ATPase (excluding Na-ATPase) & $v_{\text {ATPase }}^{n}=V_{\max , A T P a s e}^{n} \cdot\left[\frac{A T P_{n}}{\operatorname{ATP}_{n}+K_{m, A T P}}\right]$ \\
\hline \multicolumn{3}{|c|}{ Astrocytic metabolism } \\
\hline 10 & Hexokinase & $v_{H K}^{g}=k_{H K}^{g} \cdot A T P_{g} \cdot\left[\frac{G L C_{g}}{G L C_{g}+K_{m, G L C}}\right] \cdot[1-f(G 6 P g, 0.6,20)]$ \\
\hline 11 & Phosphoglucose isomerase & $v_{P G 1}^{g}=v_{\max , f, P G 1}^{g} \cdot\left[\frac{G 6 P_{g}}{G 6 P_{g}+K_{m, G G P}}\right]-v_{\max , r, P G 1}^{g} \cdot\left[\frac{F 6 P_{g}}{F 6 P_{g}+K_{m, F G P}}\right]$ \\
\hline 12 & Phosphofructokinase & $v_{P F K}^{g}=k_{P F K}^{g} \cdot A T P_{g}\left[1+\left(\frac{A T P_{g}}{K_{I, A T P}}\right)^{n H}\right]^{-1} \cdot\left[\frac{F 6 P_{g}}{F 6 P_{g}+K_{m, F G P}}\right]$ \\
\hline 13 & Phosphoglycerate kinase & $v_{P G K}^{g}=k_{P G K}^{g} \cdot G A P_{g} \cdot A D P_{g}\left[\frac{N A D_{g}}{N A D h_{g}}\right]$ \\
\hline 14 & Pyruvate kinase & $v_{P K}^{g}=k_{P K}^{g} \cdot P E P_{g} \cdot A D P_{g}$ \\
\hline 15 & Mitochondrial oxidation of pyruvate & $v_{m i t o}^{g}=v_{\text {max }, \text { mito }}^{g} \cdot\left[\frac{P Y R_{g}}{P Y R_{g}+K_{m, P Y R}}\right] \cdot\left[\frac{A D P_{g}}{A D P_{g}+K_{m, A D P}}\right] \cdot\left[\frac{O 2_{g}}{O 2_{g}+K_{m, O 2}}\right] \cdot\left[1-f\left(\frac{A T P_{g}}{A D P_{g}}, 20,5\right)\right.$ \\
\hline 17 & Lactate dehydrogenase & $\begin{array}{l}v_{L D H}^{g}=k_{L D H, f}^{g} \cdot P Y R_{g} \cdot N A D H_{g}-k_{L D H, r}^{g} \cdot L A C_{g} \cdot N A D_{g} \\
v_{C K}^{g}=k_{C K, f}^{g} \cdot P C r_{g} \cdot A D P_{g}-k_{C K, r}^{g} \cdot C r_{g} \cdot A T P_{g}\end{array}$ \\
\hline 18 & ATPase (excluding Na-ATPase) & $v_{C K}^{g}=V_{\max , A T P a s e}^{g} \cdot\left[\frac{A T P_{g}}{A T P_{g}+K_{m, A T P}}\right]$ \\
\hline Ader & $\begin{array}{l}\text { kinase equilibrium (neurons and astrocytes) } \\
\qquad A D P=\frac{A T P}{2} \cdot\left[-q_{A K}+\sqrt{u}\right] \\
\frac{d A M P}{d A T P}=-1+\frac{q_{A K}}{2}-0.5 \cdot \sqrt{u}+q_{A K} \cdot \frac{A N P}{A T P \cdot \sqrt{u}}\end{array}$ & with $u=q_{A K}^{2}+4 \cdot q_{A K}\left(\frac{A N P}{A T P}-1\right)$ \\
\hline
\end{tabular}

depending on the energetic requirements of the tissue and time of the day. Levels of glycogen up to $4.2 \mathrm{mMol}$ are reported for astrocytes (Brown and Ransom, 2007; units adjusted for consistency). The pool of glycogen in astrocytes is thus not negligible and could potentially sustain cerebral activity for a few minutes. Qualitatively, it would seem that under resting conditions, glycogen storage (up to $4.2 \mathrm{mMol}$ ) would be favoured, as glucose is available from the extracellular space and glycolytic requirements are lower. During high activity periods (or

Fig. 8 Stimulation and astrocytes-neurons coordination

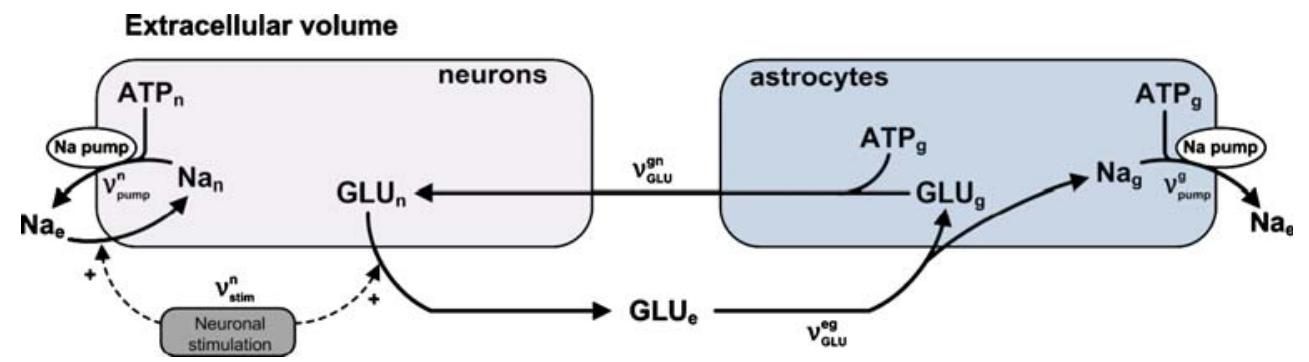




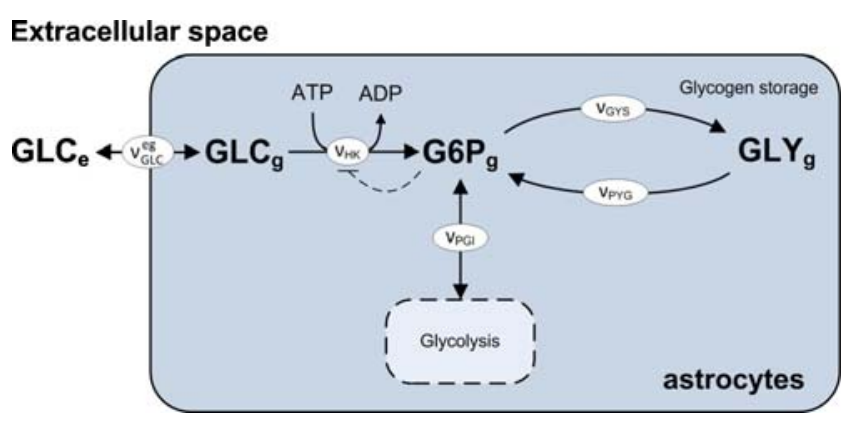

Fig. 9 Glycogen storage system

hypoglycemia), glycogen breakdown can be initiated by neurotransmitters (noradrenaline) to induce glycogenolysis. However, as was recently reported in a special issue of Glia (Vol. 55, No.12, 2007) the role of astrocytic glycogen in brain energy metabolism is much more complex than that if a simple energy reserve. The following hypotheses and mechanisms are considered in the glycogen storage system Fig. 9:

- Glycogen synthesis from G6P is described in one reaction, the glycogen synthase (vg GYS) and breakdown is described also in one reaction, the glycogen phosphorylase (vg PYG).

- Michaelis-Menten kinetics are used to describe both reactions

- GLY accumulation will be favoured when the entering flux of GLC exceeds the glycolytic requirements (i.e. at rest). However, GLY cannot be accumulated at concentrations higher than $4.2 \mathrm{mM}$.

- Upon neuronal activation, a 'signal' (noradrenaline) is assumed to induce GLY breakdown. This is modelled as a fractional increase of $\mathrm{vg}$ PYG for a certain time following the stimulation. This signal, as was the case for the stimulatory signal, can vary depending on the stimulation. This signal is, however, assumed to start after neuronal stimulation onset (delay).

- Inhibition of vg HK by its product, G6P, is considered in the model (see equation 10 in Table A.2.2). Thus, if GLY breakdown increase the G6P pool, an inhibition of vg
HK can occur, although the overall glycolytic flux would be maintained through GLY consumption. This would allow astrocytes to switch from 'pure' GLC usage to a mix of GLC and GLY usage.

The model we present here for glycogen storage considers the dynamic, regulatory response of the glycogen pool and its integration in brain energy metabolism. The last hypothesis is critical as regards the role of GLY in energy metabolism. Switching from 'pure' glucose usage to a mix of glycogen and glucose usage during high activity periods would have a critical effect on the 'energy balance' in astrocytes. Glycogenolysis has a higher energetic yield (initiating glycolytic flux with G6P instead of GLC temporarily saves one molecule of ATP). Thus, during low demand periods, the glycogen pool can be replenished, as the glycolytic requirements are lower and this would later constitute a reserve of substrate with a higher energetic yield for high demand periods. The glycogen pool must thus be viewed not only as a substrate reserve, but also as a dynamic energy reserve. Using the glycogenolytic potential in astrocytes (even for normal cerebral activity) would allow astrocytes to buffer their energy budget between high and low demand periods. Moreover, glycogen dynamics are important for the ANLS theory. As reviewed by Pellerin et al. (2007) the GLY 'dynamic usage' hypothesis is not contradictory with the ANLS. Transferring the 'energetic potential' of astrocytic GLY to neurons could be done through LAC shuttling. But the fact that astrocytes can switch to GLY usage during high demand period also potentially leaves more GLC for neurons to consume. Thus, a precise quantification of glycogen dynamics and its integration in models for brain energy metabolism is going to be crucial for future developments (Pellerin et al. 2007) Table 5, 6 and 7.

\section{A.5 The complete model}

Figure 10 (next page) presents the complete model used in this study.
Table 5 Stimulation related variables

\begin{tabular}{llll}
\hline Variable & Steady-state value & Differential equation \\
\hline $\begin{array}{lll}\text { Neuronal states } \\
\text { Nan }\end{array}$ & Sodium & 15 & $\frac{\partial N a_{n}}{\partial t}=v_{\text {leak,Na }}^{n}-3 \cdot v_{\text {pump }}^{n}+v_{\text {stim }}^{n}$ \\
GLUn & Glutamate & 3 & $\frac{\partial G L U_{n}}{\partial t}=v_{G L U}^{g n}-R_{N a-G L U} \cdot v_{\text {stim }}^{n}$ \\
Astrocytic states & Sodium & 15 & $\frac{\partial N a_{g}}{\partial t}=v_{\text {leak, Na }}^{g}-3 \cdot v_{\text {pump }}^{g}+v_{\text {stim }}^{g}$ \\
Nag & Glutamate & $1 \mathrm{e}-6$ & $\frac{\partial G L U_{g}}{\partial t}=v_{G L U}^{\text {eg }}-v_{G L U}^{g n}$ \\
GLUg & Sodiumq & 150 & $\operatorname{constant}$ \\
Extracellular states & & $\frac{\partial G L U_{e}}{\partial t}=R_{n e} \cdot R_{N a-G L U} \cdot v_{s t i m}^{n}-v_{s t i m}^{n}$ \\
Nae & Glutamate & $1 \mathrm{e}-6$ &
\end{tabular}


Table 6 Kinetic equations for stimulation related fluxes

\begin{tabular}{|c|c|c|}
\hline No. & Reaction & Kinetic equation \\
\hline \multicolumn{3}{|c|}{ Neuronal metabolism } \\
\hline 1 & Sodium leak current & $v_{\text {Leak-Na }}^{n}=\frac{S_{m} \cdot g_{N a}^{n}}{V_{n} \cdot F}\left[\frac{R \cdot T}{F} \ln \left(\frac{N a_{e}}{N a_{n}}-V m\right)\right]$ \\
\hline 2 & Neuronal $\mathrm{Na}$ inflow due to stimulation & $v_{\text {stim }}^{n}=v_{n}^{1}+\left[v_{n}^{2} \cdot{\frac{t}{t_{\text {stim }}}}^{e-t} \cdot\left(t_{\text {stim }}\right)\right] \quad$ For $0 \leq \mathrm{t} \leq \mathrm{t}_{\text {end }} *$ \\
\hline 3 & Na-ATPases & $v_{\text {pump }}^{n}=\frac{S_{m}}{V_{n}} \cdot k_{\text {pump }} \cdot A T P_{n} \cdot N a_{n} \cdot\left(1+\frac{A T P_{n}}{K_{m, N a-p u m p}}\right)^{-1}$ \\
\hline 4 & Glutamate release & $v_{G L U}^{n e}=v_{s t i m}^{n} \cdot R_{N a-G L U}$ \\
\hline \multicolumn{3}{|c|}{ Astrocytic metabolism } \\
\hline 5 & Glutamate uptake & $v_{G L U}^{e g}=v_{\max , G L U}^{e g}\left[\frac{G L U_{e}}{G L U_{e}+K_{m, G L U}}\right]$ \\
\hline 6 & Glutamate transfer to neurons & $v_{G L U}^{g n}=v_{\max , G L U}^{g n}\left[\frac{G L U_{g}}{G L U_{g}+K_{m, G L U}}\right] \cdot\left[\frac{A T P_{g}}{A T P_{g}+K_{m, A T P}}\right]$ \\
\hline 7 & Sodium leak current & $v_{\text {Leak-Na }}^{g}=\frac{S_{m} \cdot g_{N a}^{g}}{V_{g} \cdot F}\left[\frac{R \cdot T}{F} \ln \left(\frac{N a_{e}}{N a_{g}} V m\right)\right]$ \\
\hline 8 & Na-ATPases & $v_{\text {pump }}^{g}=\frac{S_{m}}{V_{g}} \cdot k_{\text {pump }} \cdot A T P_{g} \cdot N a_{g} \cdot\left(1+\frac{A T P_{g}}{K_{m, N a-p u m p}}\right)^{-1}$ \\
\hline
\end{tabular}

* ' $\mathrm{t}$ ' is time post-stimulation and tend is the duration of neuronal stimulation. Outside the period [0... tend], $\mathrm{v}^{\mathrm{n}}$ stim $=0$.

\section{A.6 Model parameters}

Table A.6.1 presents model kinetic parameters. These parameters were found through model calibration (see section on model calibration) with in vivo data on energy metabolism (see article) and brain physiology data (typical fluxes and ratios, see Appendix B). Table A.6.2 presents the physical constants and known concentrations that are used in the model. Table A.6.3 presents the 'input' parameters that are used to define the effect of neuronal stimulation.

\section{A.7 Switch function}

The present model needs to describe physiological changes in behaviour (changes in CBF during stimulation etc.) which are not well described by 'pure' on/off switches.
Sigmoid behaviour is much more common with biological systems. The following function can be used to describe a sigmoid switch:

$f(t, \delta, a)=\frac{1}{1+e^{-a \cdot(t-\delta)}}$

where ' $\mathrm{t}$ ' is the time, ' $\delta$ ' is the time of the 'event' inducing switch in behaviour and ' $\alpha$ ' is the sharpness of the change or the 'slope' during the change in behaviour. Figure 11 presents profiles of that function.

Thus, by combining different forms of that function, it is possible to describe 'smooth' or 'sharp' changes in biological behaviour without having to use binary switching. This approach allows a better description of biological behaviours and reduces discontinuities, which is important in numerical solvers for differential equations. The switch

Table 7 Glycogen storage system

\begin{tabular}{|c|c|c|c|}
\hline Variable & & Steady-state value & Differential equation \\
\hline \multicolumn{4}{|c|}{ Astrocytic state } \\
\hline GLYg & Glycogen & 3 & $\frac{\partial G L Y_{g}}{\partial t}=v_{G L Y S}^{g}-v_{G L Y P}^{g}$ \\
\hline \multicolumn{4}{|c|}{ Kinetic equations } \\
\hline 1 & Glycogen synthase & & $v_{G L Y S}^{g}=v_{\max , G L Y S}^{g} \cdot\left[\frac{G 6 P_{g}}{G 6 P_{g}+K_{m, G G P}}\right] \cdot\left[1-f\left(G L Y_{g}, 4.2,20\right)\right]$ \\
\hline 2 & Glycogen phosphorylase & & $v_{G L Y S}^{g}=v_{\max , G L Y P}^{g} \cdot\left[\frac{G 6 P_{g}}{G 6 P_{g}+K_{m, G 6 P}}\right] \cdot\left[1+f_{G L Y}\right]$ \\
\hline 3 & GLY regulation & & $f_{G L Y}=\Delta_{G L Y} \cdot\left[f\left(t, t_{0, G L Y}, 4\right)-f\left(t, t_{0, G L Y}+t_{\text {end }, G L Y}, 4\right)\right]$ \\
\hline
\end{tabular}

* ' $\mathrm{t}$ ' is time post-stimulation, $\mathrm{t} 0, \mathrm{GLY}$ is the delay (post-stimulation) before GLY breakdown starts and tend,GLY is the duration of GLY breakdown. The function $\mathrm{f}(\mathrm{t}, \delta, \mathrm{a})$ is presented in section A.7 


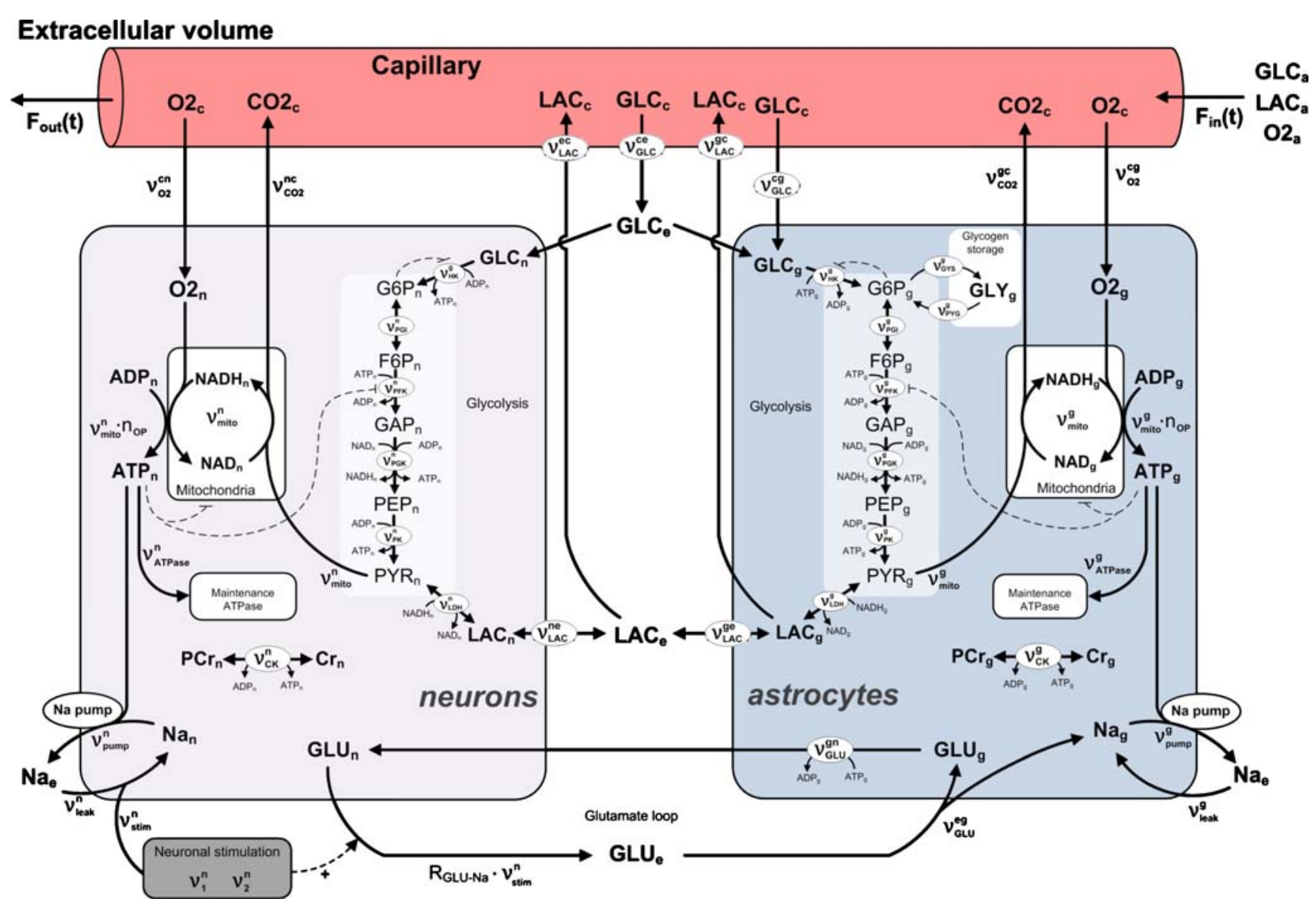

Fig. 10 Presents the complete model used in this study

can also be used with concentrations (instead of time) to represent metabolic regulation (i.e. maximum accumulation levels, inhibitions etc.) Tables 8, 9, 10.

\section{Appendix B: Model calibration with fluxes and ratios from literature}

An important consideration in building a realistic model for brain energy metabolism is the overall balancing of substrates consumption. Typical rates for GLC and O2 consumption at rest and during stimulation are presented in Figure 12.

The glutamate cycling, induced by neuronal stimulation, is characterized by the circulation of GLU in astrocytes (vgn GLU, as described, for example, in Hyder et al. 2006). The profile for vgn GLU produced by the model (Figure B-1a) is within the ranges reported in the literature (see Hyder et al. 2006). The most relevant glycolytic rate here is assumed to be the PFK rate (Figure B-1b), as it accounts for substrate coming from both GLC and GLY (see Appendix A.4 and Figure A-5 for further details). Steady-state consumption fluxes for GLC and O2 are within the ranges reported in the literature for overall
GLC and $\mathrm{O} 2$ consumption by the brain. Using the glycolytic rates (vn PFK + vg PFK) at rest and during stimulations, our model shows overall GLC consumption rates in the range of 90 to $105 \mathrm{~g}$ per day (Figure B-1f). The $\mathrm{O} 2$ consumption rate and LAC excretion by the tissue would also be in ranges described in the literature since

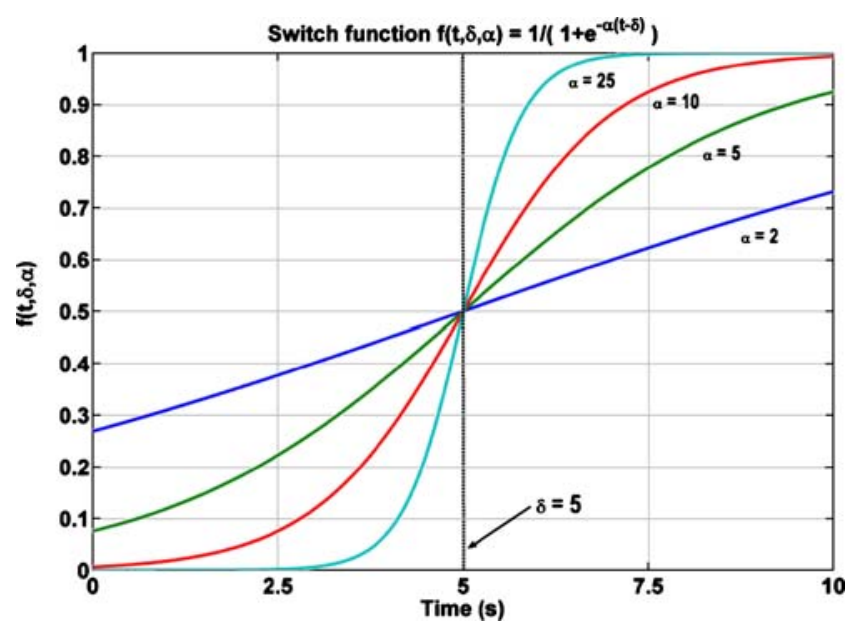

Fig. 11 Switch function behaviour 
Table 8 Model parameters

\begin{tabular}{|c|c|c|c|}
\hline Parameter name & Value & Description & Units \\
\hline $\mathrm{V}_{\max , \mathrm{glu}}^{\mathrm{gn}}$ & 0.2994 & reaction rate constant for glutamine synthase & $\mathrm{mM} \cdot \mathrm{s}^{-1}$ \\
\hline$V_{\text {max,glu }}^{\text {eg }}$ & 0.2082 & maximum uptake rate of GLU in astrocytes & $\mathrm{mM} \cdot \mathrm{s}^{-1}$ \\
\hline $\mathrm{K}_{\mathrm{m}, \mathrm{ATP}}$ & 0.0153 & affinity constant for ATP & $\mathrm{mM}$ \\
\hline $\mathrm{V}^{\mathrm{en}}{ }_{\max , \mathrm{GLC}}$ & 0.504 & maximum transport rate of GLC e->n & $\mathrm{mM} \cdot \mathrm{s}^{-1}$ \\
\hline $\mathrm{V}_{\max , \mathrm{GLC}}^{\mathrm{eg}}$ & 0.038 & maximum transport rate of glc $\mathrm{e}->\mathrm{g}$ & $\mathrm{mM} \cdot \mathrm{s}^{-1}$ \\
\hline $\mathrm{V}_{\max , \mathrm{GLC}}{ }^{\mathrm{cg}}$ & 0.010 & maximum transport rate of $\mathrm{glc} \mathrm{c}->\mathrm{g}$ & $\mathrm{mM} \cdot \mathrm{s}^{-1}$ \\
\hline$V_{\max , H K}{ }^{n}$ & 0.051 & maximum reaction rate for for $\mathrm{HK}$ in neurons & $\mathrm{mM} \cdot \mathrm{s}^{-1}$ \\
\hline $\mathrm{V}_{\max , \mathrm{HK}}{ }^{\mathrm{g}}$ & 0.050 & maximum reaction rate for for $\mathrm{HK}$ in astrocytes & $\mathrm{mM} \cdot \mathrm{s}^{-1}$ \\
\hline$V_{\text {max,f,PGI }}{ }^{n}$ & 0.502 & maximum reaction rate for for PGI in neurons & $\mathrm{mM} \cdot \mathrm{s}^{-1}$ \\
\hline $\mathrm{V}_{\max , f, P G I}{ }^{\mathrm{g}}$ & 0.483 & max. reaction rate for for PGI in astrocytes & $\mathrm{mM} \cdot \mathrm{s}^{-1}$ \\
\hline $\mathrm{V}_{\text {max,r,PGI }}{ }^{\mathrm{n}}$ & 0.503 & max. reverse reaction rate for for PGI in neurons & $\mathrm{mM} \cdot \mathrm{s}^{-1}$ \\
\hline $\mathrm{V}_{\max , \mathrm{r}, \mathrm{PGI}}{ }^{\mathrm{g}}$ & 0.451 & max. reverse reaction rate for for PGI in astrocytes & $\mathrm{mM} \cdot \mathrm{s}^{-1}$ \\
\hline $\mathrm{k}_{\mathrm{PFK}}^{\mathrm{n}}$ & 0.558 & reaction rate constant for PFK in neurons & $\mathrm{mM}^{-1} \cdot \mathrm{s}^{-1}$ \\
\hline $\mathrm{k}_{\mathrm{PFK}}{ }^{\mathrm{g}}$ & 0.403 & reaction rate constant for PFK in astrocytes & $\mathrm{mM}^{-1} \cdot \mathrm{s}^{-1}$ \\
\hline $\mathrm{k}_{\mathrm{PGK}}{ }^{\mathrm{n}}$ & 0.429 & reaction rate constant for PGK in neurons & $\mathrm{mM}^{-1} \cdot \mathrm{s}^{-1}$ \\
\hline $\mathrm{k}_{\mathrm{PGK}}^{\mathrm{g}}$ & 0.251 & reaction rate constant for PGK in astrocytes & $\mathrm{mM}^{-1} \cdot \mathrm{s}^{-1}$ \\
\hline $\mathrm{k}_{\mathrm{PK}}^{\mathrm{n}}$ & 8.61 & rate constant for $\mathrm{PK}$ in neurons & $\mathrm{mM}^{-1} \cdot \mathrm{s}^{-1}$ \\
\hline $\mathrm{kgPK}$ & 2.73 & rate constant for $\mathrm{PK}$ in astrocytes & $\mathrm{mM}^{-1} \cdot \mathrm{s}^{-1}$ \\
\hline $\mathrm{k}_{\mathrm{LDH}, \mathrm{f}}^{\mathrm{n}}$ & 5. 30 & rate constant for $\mathrm{LDH}$ in neurons & $\mathrm{mM}^{-1} \cdot \mathrm{s}^{-1}$ \\
\hline $\mathrm{k}_{\mathrm{LDH}, \mathrm{f}^{\mathrm{g}}}^{\mathrm{g}}$ & 6.26 & rate constant for $\mathrm{LDH}$ in astrocytes & $\mathrm{mM}^{-1} \cdot \mathrm{s}^{-1}$ \\
\hline $\mathrm{k}_{\mathrm{LDH}, \mathrm{r}}^{\mathrm{n}}$ & 0.105 & reverse rate constant for $\mathrm{LDH}$ in neurons & $\mathrm{mM}^{-1} \cdot \mathrm{s}^{-1}$ \\
\hline $\mathrm{k}_{\mathrm{LDH}, \mathrm{r}}^{\mathrm{g}}$ & 0.547 & reverse rate constant for $\mathrm{LDH}$ in astrocytes & $\mathrm{mM}^{-1} \cdot \mathrm{s}^{-1}$ \\
\hline $\mathrm{V}_{\max , \text { LAC }}{ }^{\mathrm{ne}}$ & 0.1978 & rate constant for lactate exchange $\mathrm{n}<->\mathrm{e}$ & $\mathrm{mM} \cdot \mathrm{s}^{-1}$ \\
\hline $\mathrm{V}_{\max , \mathrm{LAC}}{ }^{\mathrm{ge}}$ & 0.0861 & rate constant for lactate exchange $\mathrm{g}<->\mathrm{e}$ & $\mathrm{mM} \cdot \mathrm{s}^{-1}$ \\
\hline $\mathrm{V}_{\max , \mathrm{GLC}}{ }^{\mathrm{ce}}$ & 0.0496 & maximum transport rate of glc $\mathrm{c}->\mathrm{e}$ & $\mathrm{mM} \cdot \mathrm{s}^{-1}$ \\
\hline $\mathrm{V}_{\max , \mathrm{LAC}}{ }^{\mathrm{gc}}$ & 0.0002 & rate constant for lactate exchange $\mathrm{g}<->\mathrm{c}$ & $\mathrm{mM} \cdot \mathrm{s}^{-1}$ \\
\hline $\mathrm{V}_{\max , \text { LAC }}{ }^{\mathrm{ec}}$ & 0.0325 & rate constant for lactate exchange $\mathrm{e}<->\mathrm{c}$ & $\mathrm{mM} \cdot \mathrm{s}^{-1}$ \\
\hline $\mathrm{V}_{\text {max }, \text { mito }}{ }^{\mathrm{n}}$ & 0.0556 & maximum reaction rate for mitochondrial resp. & $\mathrm{mM} \cdot \mathrm{s}^{-1}$ \\
\hline $\mathrm{V}_{\text {max,mito }}{ }^{\mathrm{g}}$ & 0.0084 & maximum reaction rate for mitochondrial resp. & $\mathrm{mM} \cdot \mathrm{s}^{-1}$ \\
\hline$V_{\text {max,ATPase }}{ }^{n}$ & 0.0489 & ATPase maximum rate neurons & $\mathrm{mM} \cdot \mathrm{s}^{-1}$ \\
\hline $\mathrm{V}_{\text {max,ATPase }}{ }^{\mathrm{g}}$ & 0.0357 & ATPase maximum rate in astrocytes & $\mathrm{mM} \cdot \mathrm{s}^{-1}$ \\
\hline $\mathrm{k}_{\mathrm{CK}, \mathrm{f}}^{\mathrm{n}}$ & 0.0524 & rate constant for $\mathrm{CK}$ & $\mathrm{mM}^{-1} \cdot \mathrm{s}^{-1}$ \\
\hline $\mathrm{k}_{\mathrm{CK}, \mathrm{f}} \mathrm{g}^{\mathrm{g}}$ & 0.0243 & rate constant for $\mathrm{CK}$ & $\mathrm{mM}^{-1} \cdot \mathrm{s}^{-1}$ \\
\hline $\mathrm{k}_{\mathrm{CK}, \mathrm{r}}^{\mathrm{n}}$ & 0.0152 & reverse rate constant for $\mathrm{CK}$ & $\mathrm{mM}^{-1} \cdot \mathrm{s}^{-1}$ \\
\hline $\mathrm{k}_{\mathrm{CK}, \mathrm{r}}^{\mathrm{g}}$ & 0.0207 & reverse rate constant for $\mathrm{CK}$ & $\mathrm{mM}^{-1} \cdot \mathrm{s}^{-1}$ \\
\hline $\mathrm{V}_{\text {max,GYS }}$ & $1.53 \times 10-4$ & Maximum reaction rate for GLY synthase & $\mathrm{mM} \cdot \mathrm{s}^{-1}$ \\
\hline $\mathrm{V}_{\text {max,PYG }}$ & $4.92 \times 10-5$ & Maximum reaction rate for GLY phosphorylase & $\mathrm{mM} \cdot \mathrm{s}^{-1}$ \\
\hline $\mathrm{K}_{\mathrm{I}, \mathrm{ATP}}$ & 0.7595 & inhibition constant for ATP & $\mathrm{mM}$ \\
\hline $\mathrm{K}_{\mathrm{m}, \text { Na-pump }}$ & 0.4243 & affinity constant for Na-ATPase pump & $\mathrm{mM}$ \\
\hline $\mathrm{K}_{\mathrm{m}, \mathrm{ADP}}$ & 0.00107 & affinity constant of mito. for ADP & $\mathrm{mM}$ \\
\hline $\mathrm{K}_{\mathrm{m}, \mathrm{O} 2}$ & 0.00297 & affinity constant of mito. for $\mathrm{O} 2$ & $\mathrm{mM}$ \\
\hline $\mathrm{K}_{\mathrm{O} 2}$ & 0.0897 & $\mathrm{O} 2$ transport constant & $\mathrm{mM}$ \\
\hline $\mathrm{K}_{\mathrm{T}, \mathrm{LAC}}{ }^{\mathrm{ec}}$ & 0.764 & affinity constant for lactate exchange $\mathrm{e}<->\mathrm{c}$ & $\mathrm{mM}$ \\
\hline $\mathrm{K}_{\mathrm{T}, \mathrm{LAC}}{ }^{\mathrm{gc}}$ & 0.128 & affinity constant for lactate exchange $\mathrm{g}<->\mathrm{c}$ & $\mathrm{mM}$ \\
\hline $\mathrm{K}_{\mathrm{T}, \mathrm{LAC}}{ }^{\text {ne }}$ & 0.093 & affinity constant for lactate exchange $\mathrm{n}<->\mathrm{e}$ & $\mathrm{mM}$ \\
\hline $\mathrm{K}_{\mathrm{T}, \mathrm{LAC}}{ }^{\mathrm{ge}}$ & 0.221 & affinity constant for lactate exchange $\mathrm{g}<->\mathrm{e}$ & $\mathrm{mM}$ \\
\hline $\mathrm{K}_{\mathrm{T}, \mathrm{GLC}}^{\mathrm{ce}}$ & 8.45 & affinity constant of glc transport $\mathrm{c}->\mathrm{e}$ & $\mathrm{mM}$ \\
\hline $\mathrm{K}_{\mathrm{T}, \mathrm{GLC}}^{\mathrm{cg}}$ & 9.92 & affinity constant of astrocytes for GLC from cap. & $\mathrm{mM}$ \\
\hline $\mathrm{K}_{\mathrm{T}, \mathrm{GLC}}{ }^{\text {en }}$ & 5.32 & affinity constant of neurons for GLC & $\mathrm{mM}$ \\
\hline
\end{tabular}




\begin{tabular}{|c|c|c|c|}
\hline $\mathrm{K}_{\mathrm{T}, \mathrm{GLC}}{ }^{\mathrm{eg}}$ & 3.52 & affinity constant of astrocytes for GLC & $\mathrm{mM}$ \\
\hline $\mathrm{K}_{\mathrm{m}, \mathrm{GLC}}$ & 0.105 & affinity for intracellular GLC & $\mathrm{mM}$ \\
\hline $\mathrm{PS}_{\mathrm{cap}}^{\mathrm{n}}$ & 0.220 & $\mathrm{O}_{2}$ mass transfer constant between cap. and neuron & $\mathrm{s}^{-1}$ \\
\hline $\mathrm{PS}_{\text {cap }}^{\mathrm{g}}$ & 0.245 & $\mathrm{O}_{2}$ mass transfer constant between cap. and astrocyte & $\mathrm{s}^{-1}$ \\
\hline $\mathrm{K}_{\mathrm{m}, \mathrm{G} 6 \mathrm{P}}$ & 0.50 & affinity for G6P & $\mathrm{mM}$ \\
\hline $\mathrm{K}_{\mathrm{m}, \mathrm{F} 6 \mathrm{P}-\mathrm{PGI}}$ & 0.06 & Affinity of PGI for F6P & $\mathrm{mM}$ \\
\hline $\mathrm{K}_{\mathrm{m}, \mathrm{F} 6 \mathrm{P}-\mathrm{PFK}}$ & 0.06 & affinity of PFK for F6P & $\mathrm{mM}$ \\
\hline $\mathrm{nH}$ & 4 & Hill coefficient for ATP inhibition & \\
\hline $\mathrm{nh}_{\mathrm{o} 2}$ & 2.7 & $\mathrm{O}_{2}$ reaction order constant & \\
\hline $\mathrm{K}_{\mathrm{m}, \mathrm{gly}}$ & 0.982 & affinity constant for GLY & $\mathrm{mM}$ \\
\hline $\mathrm{K}_{\mathrm{m}, \mathrm{pyr}}$ & 0.063 & affinity constant of mitochondria for PYR & $\mathrm{mM}$ \\
\hline $\mathrm{K}_{\mathrm{m}, \mathrm{glu}}$ & 0.05 & affinity constant for GLU & $\mathrm{mM}$ \\
\hline $\mathrm{K}_{\mathrm{m} \text {,nadh }}$ & 0.015 & affinity constant for NADH & $\mathrm{mM}$ \\
\hline $\mathrm{K}_{\mathrm{m}, \mathrm{nad}}$ & 0.069 & affinity constant for NAD & $\mathrm{mM}$ \\
\hline $\mathrm{q}_{\mathrm{AK}}$ & 0.92 & equilibrium constant for $\mathrm{AK}$ & \\
\hline
\end{tabular}

Table 9 Physical constants and known concentrations

\begin{tabular}{|c|c|c|c|}
\hline Parameter name & Value & Description & Units \\
\hline $\mathrm{PCr}_{\text {tot }}$ & 5 & total $\mathrm{PCr}+\mathrm{Cr}$ & $\mathrm{mM}$ \\
\hline $\mathrm{O} 2_{\mathrm{a}}$ & 8.34 & arterial $\mathrm{O}_{2}$ & $\mathrm{mM}$ \\
\hline $\mathrm{CO} 2_{\mathrm{a}}$ & 27.5 & arterial $\mathrm{CO}_{2}$ & $\mathrm{mM}$ \\
\hline $\mathrm{GLC}_{\mathrm{a}}$ & 4.8 & arterial GLC & $\mathrm{mM}$ \\
\hline $\mathrm{LAC}_{\mathrm{a}}$ & 0.313 & arterial LAC & $\mathrm{mM}$ \\
\hline NADHtot & 0.22 & total $\mathrm{NADH}+\mathrm{NAD}$ & $\mathrm{mM}$ \\
\hline ANP & 2.38 & total ATP + ADP + AMP & $\mathrm{mM}$ \\
\hline$t_{v}$ & 35 & characteristic time for venous vol. dynamics & s \\
\hline $\mathrm{k}_{\text {pump }}$ & $3.17 \times 10-7$ & transport rate constant & $\mathrm{cm} \cdot \mathrm{mM}^{-1} \cdot \mathrm{s}^{-1}$ \\
\hline $\mathrm{CBF}_{0}$ & 0.012 & cerebral blood flow in resting conditions & $\mathrm{s}^{-1}$ \\
\hline $\mathrm{V}_{\mathrm{e}}$ & 0.2 & extracellular fraction volume & \\
\hline $\mathrm{V}_{\mathrm{c}}$ & 0.0055 & capillary fraction volume & \\
\hline $\mathrm{V}_{\mathrm{g}}$ & 0.25 & glial fraction volume & \\
\hline$V_{n}$ & 0.45 & neuron fraction volume & \\
\hline $\mathrm{R}_{\mathrm{en}}$ & $4 / 9$ & volumetric ratio $(\mathrm{Ve} / \mathrm{Vn})$ & \\
\hline $\mathrm{R}_{\mathrm{eg}}$ & 0.8 & volumetric ratio $(\mathrm{Ve} / \mathrm{Vg})$ & \\
\hline $\mathrm{R}_{\mathrm{ce}}$ & 0.0275 & volumetric ratio $(\mathrm{Vc} / \mathrm{Ve})$ & \\
\hline $\mathrm{R}_{\mathrm{cn}}$ & 0.01222 & volumetric ratio $(\mathrm{Vc} / \mathrm{Vn})$ & \\
\hline $\mathrm{R}_{\mathrm{cg}}$ & 0.022 & volumetric ratio $(\mathrm{Vc} / \mathrm{Vg})$ & \\
\hline nOP & 15 & oxidative phosphorylation ratio (ATP produced per PYR consumed) & \\
\hline $\mathrm{N}_{\text {aero }}$ & 3 & aerobic metabolism ratio (O2 consumed per PYR) & \\
\hline $\mathrm{V}_{\mathrm{v}, 0}$ & 0.0237 & base venous volume fraction & \\
\hline $\mathrm{HbOP}$ & 8.6 & $\mathrm{O}_{2}$ concentration with hemoglobin & $\mathrm{mM}$ \\
\hline $\mathrm{Na}_{\mathrm{e}}$ & 150 & extracellular sodium & $\mathrm{mM}$ \\
\hline $\mathrm{F}$ & 96500 & molecular charge & $\mathrm{C} \cdot \mathrm{mol}^{-1}$ \\
\hline RT & 2577340 & perfect gas constant and temperature $\mathrm{kPa}$ & $\mathrm{L} \cdot \mathrm{mmol}^{-1}$ \\
\hline $\mathrm{Sm}^{\mathrm{n}}$ & 40500 & characteristic length for $\mathrm{Na}$ (neurons) & $\mathrm{cm}^{-1}$ \\
\hline $\mathrm{Sm}^{\mathrm{g}}$ & 10500 & characteristic length for $\mathrm{Na}$ (astrocytes) & $\mathrm{cm}^{-1}$ \\
\hline $\mathrm{g}_{\mathrm{Na}}{ }^{\mathrm{n}}$ & 0.0039 & surface resistance & $\mathrm{mS} \cdot \mathrm{cm}^{-2}$ \\
\hline $\mathrm{g}_{\mathrm{Na}} \mathrm{g}$ & 0.0039 & surface resistance & $\mathrm{mS} \cdot \mathrm{cm}^{-2}$ \\
\hline $\mathrm{Vm}$ & -70 & surface potential & $\mathrm{mV}$ \\
\hline
\end{tabular}


Table 10 Stimulation parameters

\begin{tabular}{llll}
\hline Parameter name & Value & Description & Units \\
$\mathrm{v}_{\mathrm{n}}{ }^{1}$ (tail pinch) & 0.041 & base sodium transport rate during stimulation & $\mathrm{mM} \cdot \mathrm{s}^{-1}$ \\
$\mathrm{v}_{\mathrm{n}}{ }^{2}$ (tail pinch) & 2.56 & early stimulation of sodium transport rate & $\mathrm{mM} \cdot \mathrm{s}^{-1}$ \\
$\mathrm{v}_{\mathrm{n}}{ }^{1}$ (restraint) & 0.027 & base sodium transport rate during stimulation & $\mathrm{mM} \cdot \mathrm{s}^{-1}$ \\
$\mathrm{v}_{\mathrm{n}}{ }^{2}$ (restraint) & 3.83 & early stimulation of sodium transport rate & $\mathrm{mM} \cdot \mathrm{s}^{-1}$ \\
$\mathrm{t}_{\text {stim }}$ (tail pinch) & 2 & time constant of stimulation & $\mathrm{s}$ \\
$\mathrm{t}_{\text {stim }}$ (restraint) & 5.2 & time constant of stimulation & $\mathrm{s}$ \\
$\mathrm{t}_{\text {end }}$ & 300 & duration of neuronal stimulation & $\mathrm{s}$ \\
$\Delta_{\mathrm{f}}$ & 0.42 & CBF fractional increase during stimulation & \\
$\mathrm{R}_{\mathrm{GLU}-\mathrm{Na}}$ & 0.075 & GLU/Na ratio for release of GLU by neurons during stimulation & \\
$\Delta_{\mathrm{GLY}}$ & 62 & GLY breakdown fractional increase during stimulation & \\
$\mathrm{t}_{0, \mathrm{GLY}}$ & 71 & delay before GLY breakdown (post-stimulation) & $\mathrm{s}$ \\
$\mathrm{t}_{\text {end,GLY }}$ & 403 & duration of GLY breakdown & $\mathrm{s}$ \\
\hline
\end{tabular}

the simulated $\mathrm{O} 2$ to GLC ratio (5.75 at rest and going down during stimulation, see Figure B-1d) is also in the range of reported values. The rate of GLC consumption (Figure B-1f) is only marginally smaller at rest $(90 \mathrm{~g} \cdot \mathrm{d}-1)$ than during neuronal activation $(100-105 \mathrm{~g} \cdot \mathrm{d}-1$ depending on stimulation parameters). This is in accordance with the literature, as a high baseline rate for GLC metabolism in the brain is the general consensus (Magistretti 2006 and references therein). Thus the model calibration, as regards the overall GLC, O2 and LAC rates (at rest and during stimulation) produced a physiological behaviour representative of the cerebral environment.

The phenomenon of predominant GLC consumption in astrocytes, with further oxidation in neurons $\left(v^{\mathrm{g}}{ }_{\text {,PFK }}>v^{\mathrm{n}}\right.$ PFK while $\mathrm{v}^{\mathrm{n}}{ }_{\text {mito }}>\mathrm{v}_{\text {mito }}{ }^{\mathrm{g}}$, as was the case in the Hyder et al. framework and in many ANLS supporting arguments) is not observed in the simulations. In our model, GLC consumption happens mostly in neurons (at rest: $\mathrm{V}_{\mathrm{PFK}}{ }^{\mathrm{n}} \approx$
Fig. 12 Simulated fluxes for GLC oxidative metabolism and O2/GLC ratio during resting and neuronal stimulation ( $5 \mathrm{~min}$ tail pinch starting at $\mathrm{t}=1000 \mathrm{~s})$.
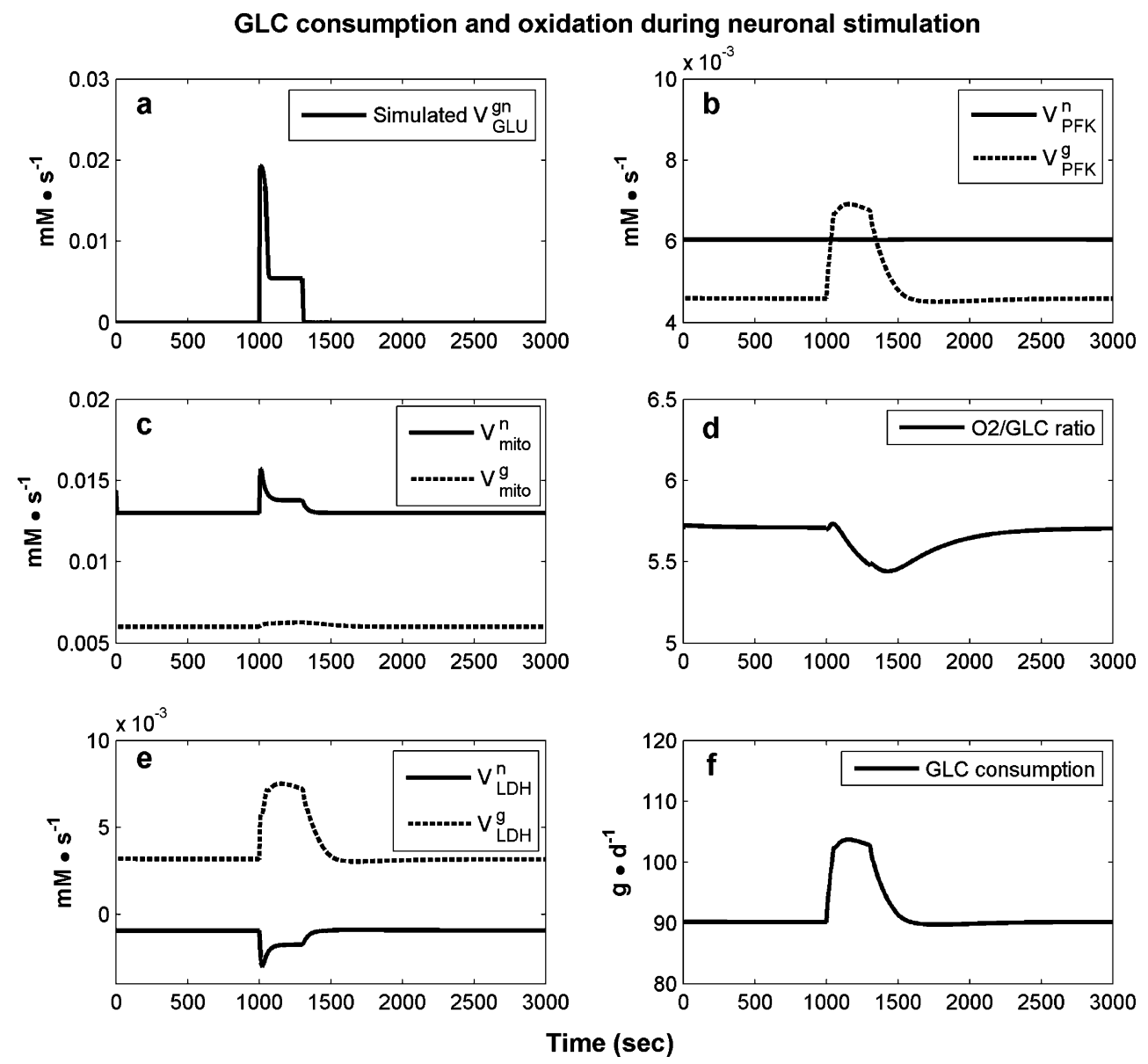
$6 \mu \mathrm{M} \cdot \mathrm{s}^{-1}$ and $\nu_{, \mathrm{PFK}}{ }^{\mathrm{g}} \approx 4.5 \mu \mathrm{M} \cdot \mathrm{s}^{-1}$, Figure B-1b). During stimulation, however, glycolytic rate in astroyctes can be slightly higher than in neurons (depending on the strength of the stimulus).

PYR oxidation is much more pronounced in neurons $\left(\mathrm{v}_{\text {mito }}{ }^{\mathrm{n}} \approx 13 \mu \mathrm{M} \cdot \mathrm{s}^{-1}\right.$ and $\mathrm{v}_{\text {mito }}{ }^{\mathrm{g}} \approx 6 \mu \mathrm{M} \cdot \mathrm{s}^{-1}$, see Figure B-1c) and this induces a slight shuttling of lactate from astrocytes to neurons (Figure B-1e, vn LDH is negative). However, glycolysis remains the major source of PYR for mitochondrial oxidation. In our model, LAC shuttling accounts only for $\approx 7 \%$ of the energetic requirements of resting neurons. This proportion rises to $\approx 12-15 \%$ during stimulations periods, which allows increases in energy demand in neurons be met by a sharp increase in mitochondrial rate rather than an increase in glycolytic flux. However, as regards the ANLS, our simulation results are not in agreement with a 'full' lactate shuttle (i.e. all the LAC produced in astrocytes being transferred to neurons for oxidation). This would lead to an $\mathrm{O} 2$ to GLC ratio of 6 (with no variations during stimulation), which is clearly not the case in both our simulations and in many reports from the literature.

\section{References}

Ainscow, E. K., \& Brand, M. D. (1999). Top-down Control Analysis of ATP Turnover, Glycolysis and Oxidative Phosphorylation in Rat Hepatocytes. European Journal of Biochemistry, 263, 671685. doi:10.1046/j.1432-1327.1999.00534.x.

Aubert, A., \& Costalat, R. (2005). Interactions between astrocytes and neurons studied using a mathematical of compartimentalized energy metabolism. Journal of Cerebral Blood Flow and Metabolism, 25, 1476-1490. doi:10.1038/sj.jcbfm.9600144.

Aubert, A., Costalat, R., Magistretti, P. J., \& Pellerin, L. (2005). Brain lactate kinetics: modeling evidence for neuronal lactate uptake upon activation. Proceedings of the National Academy of Sciences of the United States of America, 102, 16448-16453. doi:10.1073/pnas.0505427102.

Barros, L. F., Bittner, C. X., Loaiza, A., \& Porras, O. H. (2007). A Quantitative Overview of Glucose Dynamics in the Gliovascular Unit. Glia, 55, 1222-1237. doi:10.1002/glia.20375.

Bolger, F., Serra, P. A., O’Neill, R. D., Fillenz, M., \& Lowry, J. P. (2006). Real-time monitoring of brain extracellular lactate. In G. Di Chiara, E. Carboni, V. Valentini, E. Acquas, V. Bassareo \& C. Cadoni (Eds.), Monitoring Molecules in Neuroscience, pp. p286288. Cagliara, Italy: University of Cagliari Press.

Brown, A. M., Sickmann, H. M., Fosgerau, K., Lund, T. M., Schousboe, A., Waagepetersen, H. S., et al. (2005). Astrocytes glycogen metabolism is required for neuronal activity during aglycemia or intense stimulation in mouse white matter. Journal of Neuroscience Research, 79, 74-80. doi:10.1002/ jnr.20335.

Brown, A. M., \& Ransom, B. R. (2007). Astrocytes Glycogen and Brain Energy Metabolism. Glia, 55, 1263-1271. doi:10.1002/ glia.20557.

Cakir, T., Aslan, S., Saybasili, H., Akin, A., \& Ulgen, K. (2007). Reconstruction and Flux Analysis of Coupling Between Metabolic Pathways of Astrocytes and Neurons: Application to
Cerebral Hypoxia. Theoretical Biology \& Medical Modelling, 4, 48-66. doi:10.1186/1742-4682-4-48.

Fillenz, M., \& Lowry, J. P. (1998). Studies of the Source of Glucose in the Extracellular Compartment of the Rat Brain. Developmental Neuroscience, 20, 365-368. doi:10.1159/ 000017332.

Forsyth, R. J. (1996). Astrocytes and the delivery of glucose from plasma to neurons. Neurochemistry International, 28(3), 231241. doi:10.1016/0197-0186(95)00094-1.

Gjedde, A. (1997). The Relation Between Brain Function and Cerebral Blood Flow and Metabolism. In H. H. Batjer (Ed.), Cerebrovascular disease, pp. 23-40. USA, Lippinscott-Raven: Philadelphia.

Gjedde, A. (2002). Coupling of Blood Flow to Neuronal Excitability. In W. Walz (Ed.), The Neuronal Environment: Brain Homeostasis in Health and Disease, pp. 432-444. NJ, USA, Humana Press: Totowa.

Gruetter, R., Seaquist, E. R., \& Ugurbil, K. (2001). A mathematical model of compartmentalized neurotransmitter metabolism in the human brain. American Journal of Physiology. Endocrinology and Metabolism, 281, 100-112.

Haefner, J.W. (1996) Modelling Biological Systems: Principles and Applications. New York, ITP Chapman \& Hall.

Heinrich, R., \& Schuster, S.(1996) The regulation of cellular systems. New York, ITP Chapman \& Hall.

Hyder, F., Patel, A. B., Gjedde, A., Rothman, D. L., Behar, K. L., \& Shulman, R. G. (2006). Neuronal-Glial Glucose Oxidation and Glutamatergic-GABAergic Function. Journal of Cerebral Blood Flow and Metabolism, 26, 865-877. doi:10.1038/sj. jcbfm.9600263.

Lowry, J. P., \& O'Neill, R. D. (2006) Neuroanalytical chemistry in vivo using electrochemical sensors in Encyclopedia of Sensors, Grimes, C.A., Dickey, E.C. \& Pishko, M.V ed(s)., American Scientific Publishers, California, USA.

Magistretti P. J. (2006) Neuron-glia metabolic coupling and plasticity. The Journal of Experimental Biology. 209, 2304-2311.

McNay, E. C., \& Gold, P. E. (1999). Extracellular glucose concentrations in the rat hippocampus measured by zeronet-flux: effects of microdialysis flow rate, strain, and age. Journal of Neurochemistry, 72, 785-790. doi:10.1046/j.14714159.1999.720785.x.

McNay, E. C., McCarty, R. C., \& Gold, P. E. (2001). Fluctuations in Brain Glucose Concentration during Behavioral Testing: Dissociations between Brain Areas and between Brain and Blood. Neurobiology of Learning and Memory, 75(3), 325-337. doi:10.1006/nlme.2000.3976.

Miller, G. (2008). Growing pains for fMRI. Science, 320, 1412-1414. doi: $10.1126 /$ science.320.5882.1412.

Pellerin, L., \& Magistretti, P. J. (1994). Glutamate uptake into astrocytes stimulates aerobic glycolysis: A mechanism coupling neuronal activity to glucose utilization. Proceedings of the National Academy of Sciences of the United States of America, 91, 10625-10629. doi:10.1073/pnas.91.22.10625.

Pellerin, L., Bouzier-Sore, A. K., Auber, A., Serres, S., Merle, M., Costalat, R., et al. (2007). Activity-Dependant Regulation of Energy Metabolism by Astrocytes: An Update. Glia, 55, 12511262. doi:10.1002/glia.20528.

Schmidt, H., \& Jirstand, M. (2006). Systems Biology Toolbox for MATLAB: A computational platform for research in Systems Biology. Bioinformatics (Oxford, England), 22, 514-515. doi:10.1093/bioinformatics/bti799

Shen, J., Petersen, K., Behar, K., Brown, P., Nixon, T., Mason, G., et al. (1999). Determination of the rate of the glutamate/glutamine cycle in the human brain by in vivo 13C NMR. Proceedings of the National Academy of Sciences of the United States of America, 96, 8235-8240. doi:10.1073/pnas.96.14.8235. 
Shestov, A. A., Valette, J., Ugurbil, K., \& Henry, P.-G. (2007). On the reliability of ${ }^{13} \mathrm{C}$ metabolic modeling with two-compartment neuronal-glial models. Journal of Neuroscience Research, 85 (15), 3294-3303. doi:10.1002/jnr.21269.

Simpson, I. A., Carruthers, A., \& Vannucci, S. J. (2007). Supply and Demand in Cerebral Energy Metabolism: The Role of Nutrient Transporters. Journal of Cerebral Blood Flow and Metabolism, 27, 1766-1791. doi:10.1038/sj.jcbfm.9600521.

Uffmann, K., \& Gruetter, R. (2007). Mathematical modeling of ${ }^{13} \mathrm{C}$ label incorporation of the TCA cycle: The concept of composite precursor function. Journal of Neuroscience Research, 85(15), 3304-3317. doi:10.1002/jnr.21392.
Underwood, A. H., \& Newsholme, E. A. (1965). Properties of Phosphofructokinase From Rat Liver and Their Relation to the Control of Glycolysis and Gluconeogenesis. The Biochemical Journal, 95, 868-875.

Varma, A., \& Palsson, B. O. (1994). Metabolic Flux Balancing: Basic concepts, Scientific and Practical Use. Bio/Technology, 12, 994 998. doi:10.1038/nbt1094-994.

Zwingmann, C., \& Butterworth, R. (2005). An update on the role of brain glutamine synthesis and its relation to cell-specific energy metabolism in the hyperammonemic brain: Further studies using NMR spectroscopy. Neurochemistry International, 47, 19-30. doi:10.1016/j.neuint.2005.04.003. 